EUROPEAN ORGANIZATION FOR NUCLEAR RESEARCH

CERN-EP-PH/2005-015

22 March 2005

\title{
Determination of heavy quark non-perturbative parameters from spectral moments in semileptonic B decays
}

DELPHI Collaboration

\begin{abstract}
Moments of the hadronic invariant mass and of the lepton energy spectra in semileptonic B decays have been determined with the data recorded by the DELPHI detector at LEP. From measurements of the inclusive $b$-hadron semileptonic decays, and imposing constraints from other measurements on $b$ and $c$-quark masses, the first three moments of the lepton energy distribution and of the hadronic mass distribution, have been used to determine parameters which enter into the extraction of $\left|\mathrm{V}_{c b}\right|$ from the measurement of the inclusive $b$-hadron semileptonic decay width. The values obtained in the kinetic scheme are:

$$
\begin{aligned}
m_{b}(1 \mathrm{GeV}) & =4.591 \pm 0.062 \pm 0.039 \pm 0.005 \mathrm{GeV} / c^{2} \\
m_{c}(1 \mathrm{GeV}) & =1.170 \pm 0.093 \pm 0.055 \pm 0.005 \mathrm{GeV} / c^{2} \\
\mu_{\pi}^{2}(1 \mathrm{GeV}) & =0.399 \pm 0.048 \pm 0.034 \pm 0.087 \mathrm{GeV}^{2} \\
\tilde{\rho}_{D}^{3} & =0.053 \pm 0.017 \pm 0.011 \pm 0.026 \mathrm{GeV}^{3}
\end{aligned}
$$
\end{abstract}

and include corrections at order $1 / m_{b}^{3}$.

Using these results, and present measurements of the inclusive semileptonic decay partial width of $b$-hadrons at LEP, an accurate determination of $\left|\mathrm{V}_{c b}\right|$ is obtained:

$$
\left|\mathrm{V}_{c b}\right|=0.0421 \times\left(1 \pm 0.014_{\text {meas. }} \pm 0.014_{\text {fit }} \pm 0.015_{\text {th. }}\right) .
$$





T.Allmendinger ${ }^{17}$, P.P.Allport ${ }^{23}$, U.Amaldi ${ }^{29}$, N.Amapane ${ }^{45}$, S.Amato ${ }^{48}$, E.Anashkin ${ }^{36}$, A.Andreazza ${ }^{28}$, S.Andringa ${ }^{22}$, N.Anjos ${ }^{22}$, P.Antilogus ${ }^{25}$, W-D.Apel ${ }^{17}$, Y.Arnoud ${ }^{14}$, S.Ask ${ }^{26}$, B.Asman ${ }^{44}$, J.E.Augustin ${ }^{25}$, A.Augustinus ${ }^{8}$, P.Baillon ${ }^{8}$, A.Ballestrero ${ }^{46}$, P.Bambade ${ }^{20}$, R.Barbier ${ }^{27}$, D.Bardin ${ }^{16}$, G.J.Barker ${ }^{17}$, A.Baroncelli ${ }^{39}$, M.Battaglia ${ }^{8}$, M.Baubillier ${ }^{25}$, K-H.Becks ${ }^{53}$, M.Begalli ${ }^{6}$, A.Behrmann ${ }^{53}$, E.Ben-Haim ${ }^{20}$, N.Benekos ${ }^{32}$, A.Benvenuti ${ }^{5}$, C.Berat ${ }^{14}$, M.Berggren ${ }^{25}$, L.Berntzon $^{44}$, D.Bertrand ${ }^{2}$, M.Besancon ${ }^{40}$, N.Besson ${ }^{40}$, D.Bloch ${ }^{9}$, M.Blom $^{31}$, M.Bluj ${ }^{52}$, M.Bonesini ${ }^{29}$, M.Boonekamp $^{40}$, P.S.L.Booth ${ }^{23}$, G.Borisov ${ }^{21}$, O.Botner ${ }^{49}$, B.Bouquet ${ }^{20}$, T.J.V.Bowcock ${ }^{23}$, I.Boyko ${ }^{16}$, M.Bracko $^{43}$, R.Brenner ${ }^{49}$, E.Brodet ${ }^{35}$, P.Bruckman ${ }^{18}$, J.M.Brunet ${ }^{7}$, P.Buschmann ${ }^{53}$, M.Calvi ${ }^{29}$, T.Camporesi ${ }^{8}$, V.Canale ${ }^{38}$, F.Carena ${ }^{8}$, N.Castro $^{22}$, F.Cavallo ${ }^{5}$, M.Chapkin ${ }^{42}$, Ph.Charpentier ${ }^{8}$, P.Checchia ${ }^{36}$, R.Chierici ${ }^{8}$, P.Chliapnikov ${ }^{42}$, J.Chudoba ${ }^{8}$, S.U.Chung ${ }^{8}$, K.Cieslik ${ }^{18}$, P.Collins ${ }^{8}$, R.Contri ${ }^{13}$, G.Cosme ${ }^{20}$, F.Cossutti ${ }^{47}$, M.J.Costa ${ }^{50}$, D.Crennell ${ }^{37}$, J.Cuevas ${ }^{34}$, J.D'Hondt $^{2}$, J.Dalmau ${ }^{44}$, T.da Silva ${ }^{48}$, W.Da Silva ${ }^{25}$, G.Della Ricca ${ }^{47}$, A.De Angelis ${ }^{47}$, W.De Boer ${ }^{17}$, C.De Clercq ${ }^{2}$, B.De Lotto ${ }^{47}$, N.De Maria ${ }^{45}$, A.De Min ${ }^{36}$, L.de Paula ${ }^{48}$, L.Di Ciaccio ${ }^{38}$, A.Di Simone ${ }^{39}$, K.Doroba ${ }^{52}$, J.Drees ${ }^{53,8}$, G.Eigen $^{4}$, T.Ekelof ${ }^{49}$, M.Ellert ${ }^{49}$, M.Elsing ${ }^{8}$, M.C.Espirito Santo ${ }^{22}$, G.Fanourakis ${ }^{11}$, D.Fassouliotis ${ }^{11,3}$, M.Feindt ${ }^{17}$,

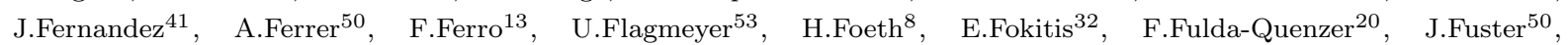
M.Gandelman ${ }^{48}$, C.Garcia ${ }^{50}$, Ph.Gavillet ${ }^{8}$, E.Gazis ${ }^{32}$, R.Gokieli ${ }^{8,52}$, B.Golob ${ }^{43}$, G.Gomez-Ceballos ${ }^{41}$, P.Goncalves ${ }^{22}$, E.Graziani ${ }^{39}$, G.Grosdidier ${ }^{20}$, K.Grzelak ${ }^{52}$, J.Guy ${ }^{37}$, C.Haag ${ }^{17}$, A.Hallgren ${ }^{49}$, K.Hamacher ${ }^{53}$, K.Hamilton ${ }^{35}$, S.Haug ${ }^{33}$, F.Hauler ${ }^{17}$, V.Hedberg ${ }^{26}$, M.Hennecke ${ }^{17}$, H.Herr ${ }^{\dagger 8}$, J.Hoffman ${ }^{52}$, S-O.Holmgren ${ }^{44}$, P.J.Holt ${ }^{8}$, M.A.Houlden ${ }^{23}$, K.Hultqvist ${ }^{44}$, J.N.Jackson ${ }^{23}$, G.Jarlskog ${ }^{26}$, P.Jarry ${ }^{40}$, D.Jeans ${ }^{35}$, E.K.Johansson ${ }^{44}$, P.D.Johansson ${ }^{44}$, P.Jonsson ${ }^{27}$, C.Joram ${ }^{8}$, L.Jungermann ${ }^{17}$, F.Kapusta ${ }^{25}$, S.Katsanevas ${ }^{27}$, E.Katsoufis ${ }^{32}$, G.Kernel ${ }^{43}$, B.P.Kersevan ${ }^{8,43}$, U.Kerzel ${ }^{17}$, B.T.King ${ }^{23}$, N.J.Kjaer ${ }^{8}$, P.Kluit ${ }^{31}$, P.Kokkinias ${ }^{11}$, C.Kourkoumelis ${ }^{3}$, O.Kouznetsov ${ }^{16}$, Z.Krumstein ${ }^{16}$, M.Kucharczyk ${ }^{18}$, J.Lamsa $^{1}$, G.Leder ${ }^{51}$, F.Ledroit ${ }^{14}$, L.Leinonen ${ }^{44}$, R.Leitner ${ }^{30}$, J.Lemonne ${ }^{2}$, V.Lepeltier ${ }^{20}$, T.Lesiak ${ }^{18}$, W.Liebig ${ }^{53}$, D.Liko ${ }^{51}$, A.Lipniacka ${ }^{44}$, J.H.Lopes ${ }^{48}$, J.M.Lopez ${ }^{34}$, D.Loukas ${ }^{11}$, P.Lutz ${ }^{40}$, L.Lyons ${ }^{35}$, J.MacNaughton ${ }^{51}$, A.Malek ${ }^{53}$, S.Maltezos $^{32}$, F.Mandl ${ }^{51}$, J.Marco ${ }^{41}$, R.Marco ${ }^{41}$, B.Marechal ${ }^{48}$, M.Margoni ${ }^{36}$, J-C.Marin ${ }^{8}$, C.Mariotti ${ }^{8}$, A.Markou ${ }^{11}$, C.Martinez-Rivero $^{41}$, J.Masik ${ }^{12}$, N.Mastroyiannopoulos ${ }^{11}$, F.Matorras ${ }^{41}$, $\quad$ C.Matteuzzi $^{29}$, F.Mazzucato ${ }^{36}$, M.Mazzucato $^{36}$, R.Mc Nulty ${ }^{23}$, C.Meroni ${ }^{28}$, E.Migliore ${ }^{45}$, W.Mitaroff ${ }^{51}$, U.Mjoernmark ${ }^{26}$, T.Moa ${ }^{44}$, M.Moch $^{17}$, K.Moenig ${ }^{8,10}$, R.Monge ${ }^{13}$, J.Montenegro ${ }^{31}$, D.Moraes ${ }^{48}$, S.Moreno ${ }^{22}$, P.Morettini ${ }^{13}$, U.Mueller ${ }^{53}$, K.Muenich ${ }^{53}$, M.Mulders ${ }^{31}$, L.Mundim ${ }^{6}$, W.Murray ${ }^{37}$, B.Muryn ${ }^{19}$, G.Myatt ${ }^{35}$, T.Myklebust ${ }^{33}$, M.Nassiakou ${ }^{11}$, F.Navarria ${ }^{5}$, K.Nawrocki ${ }^{52}$, R.Nicolaidou ${ }^{40}$, M.Nikolenko ${ }^{16,9}$, A.Oblakowska-Mucha ${ }^{19}$, V.Obraztsov ${ }^{42}$, A.Olshevski ${ }^{16}$, A.Onofre ${ }^{22}$, R.Orava ${ }^{15}$, K.Osterberg ${ }^{15}$, A.Ouraou ${ }^{40}$, A.Oyanguren ${ }^{50}$, M.Paganoni ${ }^{29}$, S.Paiano ${ }^{5}$, J.P.Palacios ${ }^{23}$, H.Palka ${ }^{18}$, Th.D.Papadopoulou ${ }^{32}$, L.Pape ${ }^{8}$, C.Parkes ${ }^{24}$, F.Parodi ${ }^{13}$, U.Parzefall ${ }^{8}$, A.Passeri ${ }^{39}$, O.Passon ${ }^{53}$, L.Peralta ${ }^{22}$, V.Perepelitsa ${ }^{50}$, A.Perrotta ${ }^{5}$, A.Petrolini ${ }^{13}$, J.Piedra ${ }^{41}$, L.Pieri ${ }^{39}$, F.Pierre ${ }^{40}$, M.Pimenta ${ }^{22}$, E.Piotto ${ }^{8}$, T.Podobnik ${ }^{43}$, V.Poireau ${ }^{8}$, M.E.Pol ${ }^{6}$, G.Polok ${ }^{18}$, V.Pozdniakov ${ }^{16}$, N.Pukhaeva ${ }^{2,16}$, A.Pullia ${ }^{29}$, J.Rames ${ }^{12}$, A.Read ${ }^{33}$, P.Rebecchi ${ }^{8}$, J.Rehn ${ }^{17}$, D.Reid ${ }^{31}$, R.Reinhardt ${ }^{53}$, P.Renton ${ }^{35}$, F.Richard ${ }^{20}$, J.Ridky ${ }^{12}$, M.Rivero ${ }^{41}$, D.Rodriguez ${ }^{41}$, A.Romero ${ }^{45}$, P.Ronchese $^{36}$, P.Roudeau ${ }^{20}$, T.Rovelli ${ }^{5}$, V.Ruhlmann-Kleider ${ }^{40}$, D.Ryabtchikov ${ }^{42}$, A.Sadovsky ${ }^{16}$, L.Salmi ${ }^{15}$, J.Salt ${ }^{50}$, C.Sander ${ }^{17}$, A.Savoy-Navarro ${ }^{25}$, U.Schwickerath ${ }^{8}$, A.Segar ${ }^{\dagger 5}$, R.Sekulin ${ }^{37}$, M.Siebel ${ }^{53}$, A.Sisakian ${ }^{16}$, G.Smadja ${ }^{27}$, O.Smirnova ${ }^{26}$, A.Sokolov ${ }^{42}$, A.Sopczak ${ }^{21}$, R.Sosnowski ${ }^{52}$, T.Spassov ${ }^{8}$, M.Stanitzki ${ }^{17}$, A.Stocchi ${ }^{20}$, J.Strauss ${ }^{51}$, B.Stugu ${ }^{4}$, M.Szczekowski ${ }^{52}$, M.Szeptycka ${ }^{52}$, T.Szumlak ${ }^{19}$, T.Tabarelli ${ }^{29}$, A.C.Taffard ${ }^{23}$, F.Tegenfeldt ${ }^{49}$, J.Timmermans ${ }^{31}$, L.Tkatchev ${ }^{16}$, M.Tobin ${ }^{23}$, S.Todorovova ${ }^{12}$, B.Tome ${ }^{22}$, A.Tonazzo ${ }^{29}$, P.Tortosa ${ }^{50}$, P.Travnicek ${ }^{12}$, D.Treille ${ }^{8}$, G.Tristram ${ }^{7}$, M.Trochimczuk ${ }^{52}$, C.Troncon ${ }^{28}$, M-L.Turluer ${ }^{40}$, I.A.Tyapkin ${ }^{16}$, P.Tyapkin ${ }^{16}$, S.Tzamarias ${ }^{11}$, V.Uvarov ${ }^{42}$, G.Valenti ${ }^{5}$, P.Van Dam ${ }^{31}$, J.Van Eldik ${ }^{8}$, N.van Remortel ${ }^{15}$, I.Van Vulpen ${ }^{8}$, G.Vegni ${ }^{28}$, F.Veloso ${ }^{22}$, W.Venus ${ }^{37}$, P.Verdier ${ }^{27}$, V.Verzi ${ }^{38}$, D.Vilanova ${ }^{40}$, L.Vitale ${ }^{47}$, V.Vrba ${ }^{12}$, H.Wahlen $^{53}$, A.J.Washbrook ${ }^{23}$, C.Weiser ${ }^{17}$, D.Wicke ${ }^{8}$, J.Wickens ${ }^{2}$, 
G.Wilkinson ${ }^{35}$, M.Winter ${ }^{9}$, M.Witek ${ }^{18}$, O.Yushchenko ${ }^{42}$, A.Zalewska ${ }^{18}$, P.Zalewski ${ }^{52}$, D.Zavrtanik ${ }^{43}$, V.Zhuravlov ${ }^{16}$, N.I.Zimin ${ }^{16}$, A.Zintchenko ${ }^{16}$, M.Zupan ${ }^{11}$

\footnotetext{
${ }^{1}$ Department of Physics and Astronomy, Iowa State University, Ames IA 50011-3160, USA

${ }^{2}$ Physics Department, Universiteit Antwerpen, Universiteitsplein 1, B-2610 Antwerpen, Belgium and IIHE, ULB-VUB, Pleinlaan 2, B-1050 Brussels, Belgium and Faculté des Sciences, Univ. de l'Etat Mons, Av. Maistriau 19, B-7000 Mons, Belgium

${ }^{3}$ Physics Laboratory, University of Athens, Solonos Str. 104, GR-10680 Athens, Greece ${ }^{4}$ Department of Physics, University of Bergen, Allégaten 55, NO-5007 Bergen, Norway

${ }^{5}$ Dipartimento di Fisica, Università di Bologna and INFN, Via Irnerio 46, IT-40126 Bologna, Italy

${ }^{6}$ Centro Brasileiro de Pesquisas Físicas, rua Xavier Sigaud 150, BR-22290 Rio de Janeiro, Brazil and Depto. de Física, Pont. Univ. Católica, C.P. 38071 BR-22453 Rio de Janeiro, Brazil and Inst. de Física, Univ. Estadual do Rio de Janeiro, rua São Francisco Xavier 524, Rio de Janeiro, Brazil ${ }^{7}$ Collège de France, Lab. de Physique Corpusculaire, IN2P3-CNRS, FR-75231 Paris Cedex 05, France ${ }^{8}$ CERN, CH-1211 Geneva 23, Switzerland

${ }^{9}$ Institut de Recherches Subatomiques, IN2P3 - CNRS/ULP - BP20, FR-67037 Strasbourg Cedex, France

${ }^{10}$ Now at DESY-Zeuthen, Platanenallee 6, D-15735 Zeuthen, Germany

${ }^{11}$ Institute of Nuclear Physics, N.C.S.R. Demokritos, P.O. Box 60228, GR-15310 Athens, Greece

${ }^{12}$ FZU, Inst. of Phys. of the C.A.S. High Energy Physics Division, Na Slovance 2, CZ-180 40, Praha 8, Czech Republic

${ }^{13}$ Dipartimento di Fisica, Università di Genova and INFN, Via Dodecaneso 33, IT-16146 Genova, Italy

${ }^{14}$ Institut des Sciences Nucléaires, IN2P3-CNRS, Université de Grenoble 1, FR-38026 Grenoble Cedex, France

${ }^{15}$ Helsinki Institute of Physics and Department of Physical Sciences, P.O. Box 64, FIN-00014 University of Helsinki, Finland

${ }^{16}$ Joint Institute for Nuclear Research, Dubna, Head Post Office, P.O. Box 79, RU-101 000 Moscow, Russian Federation

${ }^{17}$ Institut für Experimentelle Kernphysik, Universität Karlsruhe, Postfach 6980, DE-76128 Karlsruhe, Germany

${ }^{18}$ Institute of Nuclear Physics PAN,Ul. Radzikowskiego 152, PL-31142 Krakow, Poland

${ }^{19}$ Faculty of Physics and Nuclear Techniques, University of Mining and Metallurgy, PL-30055 Krakow, Poland

${ }^{20}$ Université de Paris-Sud, Lab. de l'Accélérateur Linéaire, IN2P3-CNRS, Bât. 200, FR-91405 Orsay Cedex, France

${ }^{21}$ School of Physics and Chemistry, University of Lancaster, Lancaster LA1 4YB, UK

${ }^{22}$ LIP, IST, FCUL - Av. Elias Garcia, 14-1 ${ }^{\circ}$, PT-1000 Lisboa Codex, Portugal

${ }^{23}$ Department of Physics, University of Liverpool, P.O. Box 147, Liverpool L69 3BX, UK

${ }^{24}$ Dept. of Physics and Astronomy, Kelvin Building, University of Glasgow, Glasgow G12 8QQ

${ }^{25}$ LPNHE, IN2P3-CNRS, Univ. Paris VI et VII, Tour 33 (RdC), 4 place Jussieu, FR-75252 Paris Cedex 05, France

${ }^{26}$ Department of Physics, University of Lund, Sölvegatan 14, SE-223 63 Lund, Sweden

${ }^{27}$ Université Claude Bernard de Lyon, IPNL, IN2P3-CNRS, FR-69622 Villeurbanne Cedex, France

${ }^{28}$ Dipartimento di Fisica, Università di Milano and INFN-MILANO, Via Celoria 16, IT-20133 Milan, Italy

${ }^{29}$ Dipartimento di Fisica, Univ. di Milano-Bicocca and INFN-MILANO, Piazza della Scienza 2, IT-20126 Milan, Italy

${ }^{30}$ IPNP of MFF, Charles Univ., Areal MFF, V Holesovickach 2, CZ-180 00, Praha 8, Czech Republic

${ }^{31}$ NIKHEF, Postbus 41882, NL-1009 DB Amsterdam, The Netherlands

${ }^{32}$ National Technical University, Physics Department, Zografou Campus, GR-15773 Athens, Greece

${ }^{33}$ Physics Department, University of Oslo, Blindern, NO-0316 Oslo, Norway

${ }^{34}$ Dpto. Fisica, Univ. Oviedo, Avda. Calvo Sotelo s/n, ES-33007 Oviedo, Spain

${ }^{35}$ Department of Physics, University of Oxford, Keble Road, Oxford OX1 3RH, UK

${ }^{36}$ Dipartimento di Fisica, Università di Padova and INFN, Via Marzolo 8, IT-35131 Padua, Italy

${ }^{37}$ Rutherford Appleton Laboratory, Chilton, Didcot OX11 OQX, UK

${ }^{38}$ Dipartimento di Fisica, Università di Roma II and INFN, Tor Vergata, IT-00173 Rome, Italy

${ }^{39}$ Dipartimento di Fisica, Università di Roma III and INFN, Via della Vasca Navale 84, IT-00146 Rome, Italy

${ }^{40}$ DAPNIA/Service de Physique des Particules, CEA-Saclay, FR-91191 Gif-sur-Yvette Cedex, France

${ }^{41}$ Instituto de Fisica de Cantabria (CSIC-UC), Avda. los Castros s/n, ES-39006 Santander, Spain

${ }^{42}$ Inst. for High Energy Physics, Serpukov P.O. Box 35, Protvino, (Moscow Region), Russian Federation

${ }^{43}$ J. Stefan Institute, Jamova 39, SI-1000 Ljubljana, Slovenia and Laboratory for Astroparticle Physics, Nova Gorica Polytechnic, Kostanjeviska 16a, SI-5000 Nova Gorica, Slovenia, and Department of Physics, University of Ljubljana, SI-1000 Ljubljana, Slovenia

${ }^{44}$ Fysikum, Stockholm University, Box 6730, SE-113 85 Stockholm, Sweden

${ }^{45}$ Dipartimento di Fisica Sperimentale, Università di Torino and INFN, Via P. Giuria 1, IT-10125 Turin, Italy

${ }^{46}$ INFN,Sezione di Torino and Dipartimento di Fisica Teorica, Università di Torino, Via Giuria 1, IT-10125 Turin, Italy

${ }^{47}$ Dipartimento di Fisica, Università di Trieste and INFN, Via A. Valerio 2, IT-34127 Trieste, Italy and Istituto di Fisica, Università di Udine, IT-33100 Udine, Italy

${ }^{48}$ Univ. Federal do Rio de Janeiro, C.P. 68528 Cidade Univ., Ilha do Fundão BR-21945-970 Rio de Janeiro, Brazil

${ }^{49}$ Department of Radiation Sciences, University of Uppsala, P.O. Box 535, SE-751 21 Uppsala, Sweden

${ }^{50}$ IFIC, Valencia-CSIC, and D.F.A.M.N., U. de Valencia, Avda. Dr. Moliner 50, ES-46100 Burjassot (Valencia), Spain

${ }^{51}$ Institut für Hochenergiephysik, Österr. Akad. d. Wissensch., Nikolsdorfergasse 18, AT-1050 Vienna, Austria

${ }^{52}$ Inst. Nuclear Studies and University of Warsaw, Ul. Hoza 69, PL-00681 Warsaw, Poland

${ }^{53}$ Fachbereich Physik, University of Wuppertal, Postfach 100 127, DE-42097 Wuppertal, Germany

$\dagger$ deceased
} 


\section{Introduction}

Several years ago it was proposed to obtain an accurate value of $\left|V_{c b}\right|$ by comparing the measurement of the inclusive semileptonic decay partial width in the process $b \rightarrow c \ell^{-} \bar{\nu}_{\ell}$, with the corresponding theoretical expression, obtained using the Operator Product Expansion (OPE) formalism, applied in the heavy quark mass limit [1]. A recent appraisal of the limitations of this approach can be found in [2] from which the following expression, for the semileptonic decay width, has been taken:

$$
\begin{aligned}
\Gamma_{s l}\left(b \rightarrow c \ell^{-} \bar{\nu}_{\ell}\right)= & \frac{G_{F}^{2} m_{b}^{5}(\mu)}{192 \pi^{3}}\left|V_{c b}\right|^{2}\left(1+A_{e w}\right) A^{p e r t}(r, \mu) \\
& {\left[z_{0}(r)\left(1-\frac{\left.\mu_{\pi}^{2}(\mu)-\mu_{G}^{2}(\mu)+\frac{\rho_{D}^{3}(\mu)+\rho_{L S}^{3}(\mu)}{m_{b}(\mu)}\right)}{2 m_{b}^{2}(\mu)}\right)\right.} \\
& \left.-2(1-r)^{4} \frac{\mu_{G}^{2}(\mu)-\frac{\rho_{D}^{3}(\mu)+\rho_{L S}^{3}(\mu)}{m_{b}(\mu)}}{m_{b}^{2}(\mu)}+d(r) \frac{\rho_{D}^{3}(\mu)}{m_{b}^{3}(\mu)}+\ldots\right] .
\end{aligned}
$$

In this expression, $z_{0}(r)$ is the tree-level phase space factor and $r=m_{c}^{2}(\mu) / m_{b}^{2}(\mu)$. Definitions for the other quantities are given in [2]. Equation (1) contains an expansion in $\alpha_{s}$, corresponding to perturbative QCD corrections expressed in $A^{\text {pert }}(r, \mu)$, and an expansion in $1 / m_{b}$, corresponding to non-perturbative QCD contributions. An auxiliary scale $\mu(=1 \mathrm{GeV})$ is introduced to demark the border between long- and short-distance dynamics in OPE. Numerically, $A_{e w}$, corresponding to the ultraviolet renormalization of the Fermi interaction, is well-known and amounts to $+1.4 \%[3] ; A^{\text {pert }}(r, \mu)$ corresponds to $\sim-9 \%$ corrections and the non-perturbative terms have typically few percent contributions [2]. The smallness of these last corrections comes, partly, from the fact that the term proportional to $1 / m_{b}$ is absent $[4,5]$ in Equation (1). The quantities $\mu_{\pi}^{2}, \mu_{G}^{2}, \rho_{D}^{3}$ and $\rho_{L S}^{3}$ denote the expectation values of the kinetic, chromomagnetic, Darwin and spin-orbit operators respectively. These parameters have to be determined by experiment. From the mass splitting between $\mathrm{B}^{*}$ and $\mathrm{B}$ meson the following value $\mu_{G}^{2}(1 \mathrm{GeV})=\left(0.35_{-0.02}^{+0.03}\right) \mathrm{GeV}^{2}$ has been obtained [6]. The value for $\mu_{\pi}^{2}$ is less certain; in this regularization scheme the inequality $\mu_{\pi}^{2}(\mu) \geq \mu_{G}^{2}(\mu)$ holds for any normalization scale. Constraints have been also established for terms contributing at order $1 / m_{b}^{3}$. The Darwin parameter, $\rho_{D}^{3}(\mu)$, must be positive and the spin-orbit operator value, $\rho_{L S}^{3}(\mu)$, is expected to be negative and to satisfy $-\rho_{L S}^{3} \leq \rho_{D}^{3}[2]$. In addition, it has been demonstrated that the value of $\Gamma_{s l}\left(b \rightarrow c \ell^{-} \bar{\nu}_{\ell}\right)$ is rather insensitive to the exact value of $\rho_{L S}^{3}[7]$.

A few lessons can be drawn from the previous considerations:

- the largest correction, to the naive free quark decay model, is expected to originate from perturbative QCD. Its evaluation is closely connected to the definition adopted for the running quark masses;

- in addition to the values of heavy quark masses, $m_{b}$ and $m_{c}$, two parameters need to be determined by experiment, $\mu_{\pi}^{2}$ and $\rho_{D}^{3}$, to have a control of non-perturbative QCD corrections up to the $1 / m_{b}^{3}$ order;

- the definition for the quark masses has to be consistent with both perturbative and non-perturbative dynamics.

It is then expected that the value of $\left|V_{c b}\right|$, extracted in this way from inclusive $b$ hadron semileptonic decays, can be determined with a relative uncertainty from theory 
at the $1.5 \%$ level (see [2] for a detailed breakdown of contributing sources in this number). To match this accuracy, an experimental control of the parameters governing nonperturbative QCD corrections, even at a modest level, is required and is the main purpose of the present analysis.

The determination of moments of the lepton energy and of the hadronic mass spectra in $\mathrm{B} \rightarrow \mathrm{X}_{c} \ell \bar{\nu}_{\ell}$ decays provides important information on these parameters since they can be analysed using the same formalism, and since corresponding expressions depend on the same non-perturbative parameters entering in Equation (1). The following notations for moments of the lepton energy spectrum have been used:

$$
M_{1}^{\ell}=<E_{\ell}^{*}>\text { and } M_{n}^{\prime \ell}=<\left(E_{\ell}^{*}-<E_{\ell}^{*}>\right)^{n}>, n>1
$$

where $E_{\ell}^{*}$ denotes the value of the lepton energy in the $b$-hadron rest frame; and for moments of the hadronic mass system:

$$
M_{n}^{H}=<\left(m_{H}^{2}-m_{\text {spin }}^{2}\right)^{n}>\text { and } M_{n}^{\prime H}=<\left(m_{H}^{2}-<m_{H}^{2}>\right)^{n}>.
$$

$m_{\text {spin }}=1.97375 \mathrm{GeV} / c^{2}$ denotes the spin averaged $\mathrm{D}$ meson mass, which is equal to the weighted average of the $\mathrm{D}$ and $\mathrm{D}^{*}$ masses. The OPE expresses lepton moments through quark masses as a double expansion in $\alpha_{s}$ and $1 / m_{b}$ :

$$
M_{n}^{\prime \ell}=\left(\frac{m_{b}}{2}\right)^{n}\left[\phi_{n}(r)+\bar{a}_{n}(r) \frac{\alpha_{s}}{\pi}+\bar{b}_{n}(r) \frac{\mu_{\pi}^{2}}{m_{b}^{2}}+\bar{c}_{n}(r) \frac{\mu_{G}^{2}}{m_{b}^{2}}+\bar{d}_{n}(r) \frac{\rho_{D}^{3}}{m_{b}^{3}}+\bar{s}_{n}(r) \frac{\rho_{L S}^{3}}{m_{b}^{3}}+\ldots\right] .
$$

The higher coefficient functions $\bar{b}_{n}(r), \bar{c}_{n}(r), \ldots$ are also perturbative series in $\alpha_{s}$. Due to the kinematic definition of the hadronic invariant mass, $M_{X}$, the general expression for the hadronic moments includes explicitly the value of the $b$-hadron mass, $M_{B}$ :

$$
\begin{aligned}
M_{n}^{\prime H}=m_{b}^{2 n} \sum_{l=0}\left[\frac{M_{B}-m_{b}}{m_{b}}\right]^{l}\left(E_{n l}(r)\right. & +a_{n l}(r) \frac{\alpha_{s}}{\pi}+b_{n l}(r) \frac{\mu_{\pi}^{2}}{m_{b}^{2}}+c_{n l}(r) \frac{\mu_{G}^{2}}{m_{b}^{2}} \\
& \left.+d_{n l}(r) \frac{\rho_{D}^{3}}{m_{b}^{3}}+s_{n l}(r) \frac{\rho_{L S}^{3}}{m_{b}^{3}}+\ldots\right) .
\end{aligned}
$$

Numerical values for all $r$-dependent functions entering into these expressions can be found in [7].

In the following, the $\mu$-scale independent third order correction term, $\tilde{\rho}_{D}^{3}$ has been fitted in place of $\rho_{D}^{3}$. The two quantities are related through the expression: $\tilde{\rho}_{D}^{3} \approx$ $\rho_{D}^{3}(1 \mathrm{GeV})-0.1 \mathrm{GeV}^{3}[8]$.

The consistency of the bounds set by moments of different distributions, and with other data, tests the underlying theory assumptions.

It can be noted that the approach used here does not rely on the validity of an expansion in $1 / m_{c}$, as already advocated in [7]. The value of the charm quark mass, entering into the expression of moments, is taken as a parameter whose value has also been fitted using data.

Measurements of moments have been reported by the CLEO [9-11], BaBar [12-14] and BELLE $[15,16]$ Collaborations operating at the $\Upsilon(4 S)$ resonance, and by DELPHI using preliminary data taken at the $\mathrm{Z}$ pole energy [7]. Results have also been recently published by CDF [17].

While there is an obvious advantage in measuring the energy spectra in events where the decaying $B$ rest frame almost coincides with the laboratory frame, low energy particles cannot be identified there. It is thus necessary to rely on models for extrapolating the 
lepton energy spectrum to zero energy or to resort to computations for a truncated spectrum. Performing this analysis at energies around the $\mathrm{Z}$ peak ensures sensitivity to almost the full lepton spectrum, thus reducing modelling assumptions. The main challenge at the higher energy is, for the lepton energy moments analysis, the accurate determination of the $\mathrm{B}$ rest frame.

This paper presents the results obtained from analyses of the data recorded with the DELPHI detector at LEP on moments of the hadronic mass and charged lepton energy distributions. The analysis procedures are discussed in Sections 4 and 5. In Section 6 these results are then used as inputs of a multi-parameter fit to determine the relevant corrections contributing at $\mathcal{O}\left(1 / m_{b}^{2}\right)$ and $\mathcal{O}\left(1 / m_{b}^{3}\right)$, together with the heavy quark masses. The use of higher moments guarantees a sensitivity to these parameters and the simultaneous use of the hadronic and leptonic spectra ensures that a larger number of parameters can be kept free in the fit. We discuss the results both in terms of the extraction of the parameters and the implications for $\left|V_{c b}\right|$, and as a consistency check of the underlying theoretical assumptions.

In addition, production and decay properties of broad $\mathrm{D}^{* *}$ states have been studied. They are reported in Section 3. $\mathrm{D}^{* *}$ refers, in the present analysis, to all hadronic systems of mass higher than the $\mathrm{D}^{*}$. $b$-meson semileptonic decays with charmed hadrons emitted in the final state correspond to $\overline{\mathrm{B}} \rightarrow \mathrm{D}, \mathrm{D}^{*}, \mathrm{D}^{* *} \ell^{-} \bar{\nu}_{\ell}$ transitions. $\mathrm{D}^{* *}$ states are about $30 \%$ of $b$-hadron semileptonic decays $[18,19]$. They can be resonant or nonresonant hadronic $\mathrm{D}^{(*)} n \pi$ systems. Resonant states are supposed to be dominated by $\mathrm{L}=1$, orbitally excited $c \bar{q}$ states. There are four such states: $\mathrm{D}_{0}^{*}, \mathrm{D}_{1}^{*}, \mathrm{D}_{2}^{*}$ and $\mathrm{D}_{1}$ with respectively $\mathrm{J}^{\mathrm{P}}=0^{+}, 1^{+}, 2^{+}$and $1^{+}$. The two $1^{+}$states, having common final states, mix and physical states are expected to decay into almost pure $\mathrm{D}$ or $\mathrm{S}$ wave $\mathrm{D}^{*} \pi$ or $\mathrm{D} \rho$ final states $[20]$. The two states $\left(\mathrm{D}_{2}^{*}\right.$ and $\left.\mathrm{D}_{1}\right)$ which, because of angular momentum and parity conservation, have to decay into a $\mathrm{D}$ wave are expected to be narrow and their measured widths are of the order of $20 \mathrm{MeV} / c^{2}$ [21]. The contribution from these narrow resonances has been measured $[19,22]$ and it amounts to about one third of all $\mathrm{D}^{* *}$ states. Non-resonant or broad resonant states are thus expected to have a dominant contribution in $b$-hadron semileptonic decays. In addition to $\mathrm{L}=1$ mesons, there could be states corresponding to higher angular momentum values or to radial excitations. All such states are expected to be broad [23]. Contributions from non-resonant $\mathrm{D} \pi$ or $\mathrm{D}^{*} \pi$ final states have been evaluated in [24] and this model has been considered in the present analysis.

In a previous DELPHI publication [25], production fractions of $\mathrm{D}^{* *}$ states in $b$-hadron semileptonic decays have been measured. The main focus of the present analysis is on the mass distribution of these states. This was made possible by improving the separation between signal and background events with respect to the previous analysis.

\section{Data analysis}

This study is based on $b$-hadron semileptonic decays recorded with the DELPHI detector at LEP from 1992 to 1995. Since the determination of the moments of the hadronic mass and charged lepton energy distributions have different requirements, two analyses have been performed. The first focuses on the exclusive reconstruction of $\mathrm{D}^{* *}$ states. For the second analysis a lepton sample with low background is required. This section presents those parts of the data selection and event reconstruction procedure which are common to both analyses. 


\subsection{Hadronic event selection and simulation}

In order to select hadronic Z decays, standard hadronic selection cuts have been applied. Each event has been divided into two opposite hemispheres by a plane orthogonal to the thrust axis. The polar angle ${ }^{1}$ of the thrust axis of the event had to satisfy the requirement $|\cos \theta|<0.95$. Charged and neutral particles have been clustered into jets using the LUCLUS [26] algorithm with the resolution parameter $d_{\text {join }}=5 \mathrm{GeV} / c$. About 3.4 million events have been selected from the LEP1 data sets.

The JETSET 7.3 Parton Shower [26] program has been used to generate hadronic Z decays, which were passed through the detailed detector simulation DELSIM [27] and processed by the same analysis chain as the data. A sample of about nine million $\mathrm{Z} \rightarrow q \bar{q}$ events has been used. To increase the simulation statistics, an additional sample of about 3.6 million $\mathrm{Z} \rightarrow b \bar{b}$ events, equivalent to about 17 million hadronic $\mathrm{Z}$ decays, has also been used. Statistics of the analysed hadronic samples are given in Table 1.

\begin{tabular}{|c|c|c|c|}
\hline Year & Real data & $\begin{array}{c}\text { Simulated } \\
\mathrm{Z} \rightarrow q \bar{q}\end{array}$ & $\begin{array}{c}\text { Simulated } \\
\mathrm{Z} \rightarrow b \bar{b}\end{array}$ \\
\hline $1992+1993$ & 1355805 & 3916050 & 1096199 \\
$1994+1995$ & 2012921 & 5012881 & 2495335 \\
\hline Total & 3368726 & 8928931 & 3591534 \\
\hline
\end{tabular}

Table 1: Analysed number of events. In 1992 and 1993 only two-dimensional vertex reconstruction was available.

$\mathrm{Z} \rightarrow b \bar{b}$ events have been selected using an event $b$-tagging technique [28] based on the reconstructed impact parameters of particle tracks.

Events for the exclusive analysis have been selected by requiring the presence of one tagged lepton candidate with momentum $p>2 \mathrm{GeV} / c$ and of $\mathrm{D} \mathrm{D}^{0}, \mathrm{D}^{+}$or $\mathrm{D}^{*+}$ candidate $^{2}$ in the same event hemisphere.

In order to measure moments of the lepton energy distribution in inclusive $b \rightarrow X_{c} \ell \bar{\nu}_{\ell}$ decays, events have been required to contain one tagged lepton candidate with momentum $p>2.5 \mathrm{GeV} / c$ (for muons) or $p>3 \mathrm{GeV} / c$ (for electrons).

\subsection{Muon identification}

Muons have been identified based on the response of the Muon Chambers. Details can be found in [27].

For the inclusive lepton analysis muon candidates have been accepted if they fulfilled the "standard" selection criteria, their momenta in the lab frame exceeded $2.5 \mathrm{GeV} / c$ and were contained within the polar angle intervals: $\left|\cos \theta_{\mu}\right|<0.62$ or $0.68<\left|\cos \theta_{\mu}\right|<0.94$, defining the barrel and the forward regions. The muon identification efficiency has been measured with $\mathrm{Z} \rightarrow \mu^{+} \mu^{-}$events, in the decays $\tau \rightarrow \mu \nu_{\tau} \bar{\nu}_{\mu}$ and in two-photon $\gamma \gamma \rightarrow \mu^{+} \mu^{-}$ events. A mean efficiency within the acceptance region of $0.82 \pm 0.01$ has been found, with little dependence on the muon momentum and on the track polar angle. This agrees with simulation, both in absolute value and in the momentum dependence, within a precision of $2 \%$.

\footnotetext{
${ }^{1}$ In the DELPHI coordinate system, $z$ is along the electron beam direction, $\phi$ and $\mathrm{R}$ are the azimuthal angle and radius in the $x y$ plane, and $\theta$ is the polar angle with respect to the $z$ axis.

${ }^{2}$ Throughout this paper charge-conjugate states are implicitly included.
} 
The probability for a hadron to fake a muon has been estimated on anti- $b$ tagged events. After subtracting the expected remaining muon content in this sample, the misidentification probability for hadrons has been found to be $(0.52 \pm 0.03) \%$ in the barrel and $(0.36 \pm 0.06) \%$ in the forward regions, respectively. Applying the same procedure to simulation events gave however values lower by factors of $2.03 \pm 0.12$ in the barrel and of $1.22 \pm 0.20$ in the forward regions respectively. The simulation predictions have therefore been corrected for these factors.

In the exclusive $\mathrm{D}^{* *}$ analysis, muon candidates have been accepted if they fulfilled the "loose" selection criteria and their momenta exceeded $2 \mathrm{GeV} / c$. The corresponding efficiency is $\sim 80 \%$ and the hadron misidentification probability is $\sim 1 \%$. With this selection, the correction factors to be applied to simulated events in which the candidate lepton is a misidentified hadron have been found to be $1.44 \pm 0.05(1.61 \pm 0.05)$ in 1992-93 (1994-95) data samples

\subsection{Electron identification}

Electron candidates have been tagged within the range $0.03<\left|\cos \theta_{e}\right|<0.72$ in polar angle, based on the combination of the response of the HPC, the specific ionization $d E / d x$ in the TPC and the RICH Cherenkov detector [27]. A momentum dependent cut applied on the neural net output variable, which provides a constant efficiency over the full momentum range, has been applied.

For the inclusive lepton analysis, electron candidates have been selected with a selection cut corresponding to $65 \%$ efficiency and requiring momenta greater than $3 \mathrm{GeV} / c$. The probability for a hadron to fake an electron is about $0.4 \%$. The electron identification efficiency has been measured from data by means of a sample of isolated electrons extracted from selected Compton events and one of electrons originating from photon conversions in the detector. The ratio between the efficiencies measured in data and simulated events has been parametrized as a function of the transverse momentum and polar angle of the particle track. Results are summarised in Table 2. A corresponding correction factor has been applied to simulated $q \bar{q}$ events.

The probability for mis-tagging a hadron as an electron has also been measured using data, by selecting an anti $b$-tagged background sample, as for the muons.

Electrons from photon conversions, mainly produced in the outer ID wall and in the inner TPC frame, have been rejected by removing electron candidates originating at a secondary vertex and carrying little transverse momentum relative to the direction defined from the primary to the secondary vertex. The ratio of the measured misidentification probability in data to that in simulated events is given in Table 2.

In the exclusive $\mathrm{D}^{* *}$ analysis, electron candidates have been selected with $75 \%$ efficiency and requiring momenta greater than $2 \mathrm{GeV} / c$. The probability for a hadron to fake an electron is about $1 \%$. Correction factors applied to simulated events are also given in Table 2.

\subsection{Hadronic decay reconstruction}

The reconstruction of $\mathrm{D}^{0}$ and $\mathrm{D}^{*+}$ candidates, in which the $\mathrm{D}^{0}$ decays into $\mathrm{K}^{-} \pi^{+}$, $\mathrm{K}^{-} \pi^{+} \pi^{+} \pi^{-}$or $\mathrm{K}^{-} \pi^{+}\left(\pi^{0}\right)$, is explained in detail in [29]. The reconstruction of the $\mathrm{D}^{+} \rightarrow$ $\mathrm{K}^{-} \pi^{+} \pi^{+}$decay is based on a similar approach. For all decay channels, the main steps of the analysis consist of:

- reconstructing a D decay vertex from its charged decay products; 


\begin{tabular}{|l|c|c|c|c|}
\hline & 1992 & 1993 & 1994 & 1995 \\
\hline \hline 65\% efficiency cut & & & & \\
Efficiency (data/MC) & $0.83 \pm 0.02$ & $0.83 \pm 0.02$ & $0.92 \pm 0.02$ & $0.93 \pm 0.02$ \\
\hline Misid. Prob. (data/MC) & $0.57 \pm 0.04$ & $0.77 \pm 0.05$ & $0.76 \pm 0.05$ & $0.70 \pm 0.06$ \\
\hline \hline $\begin{array}{l}75 \% \text { efficiency cut } \\
\text { Efficiency (data/MC) }\end{array}$ & $0.89 \pm 0.02$ & $0.88 \pm 0.02$ & $0.94 \pm 0.02$ & $0.94 \pm 0.02$ \\
\hline Misid. Prob. (data/MC) & $0.61 \pm 0.04$ & $0.77 \pm 0.03$ & $0.80 \pm 0.02$ & $0.74 \pm 0.03$ \\
\hline
\end{tabular}

Table 2: The ratio between values measured in real and simulated events for electron identification efficiency and probability of tagging a hadron as an electron

- selecting a mass window centred on the nominal D mass;

- reconstructing a $\mathrm{B}$ decay vertex using the $\mathrm{D}$ trajectory and a charged lepton;

- requiring a minimum distance between the $\mathrm{B}$ decay vertex and the main vertex and also between the $\mathrm{B}$ and the $\mathrm{D}$ decay vertices;

- imposing a minimum momentum on the $\mathrm{D}(6 \mathrm{GeV} / c)$ and on the $\mathrm{D}-\ell(10 \mathrm{GeV} / c)$ candidates;

- requiring a D- $\ell$ mass between 2.5 and $5.5 \mathrm{GeV} / c^{2}$.

\section{$3 \quad \mathrm{D}^{* *}$ production in $b$-hadron semileptonic decays}

In the following sections the exclusive analysis leading to the measurement of hadronic moments is presented. $\mathrm{D}^{0}, \mathrm{D}^{+}, \mathrm{D}^{*+}$ and $\mathrm{D}^{* *}$ reconstruction is described in Section 3.1. In Section 3.2 a discriminant variable that has different sensitivity to $\mathrm{D}^{* *}$ signal and background events is defined. This variable is used in Section 3.3 to measure the amount of $\mathrm{D}^{* *} \ell^{-} \bar{\nu}_{\ell}$ states in the data. From the study of the $\mathrm{D}^{* *}$ mass distribution, branching ratios and properties of different $\mathrm{D}^{* *}$ states are measured in Sections 3.4 and 3.5.

\section{1 $\mathrm{D}^{0}, \mathrm{D}^{+}, \mathrm{D}^{*+}$ and $\mathrm{D}^{* *}$ reconstruction}

Mass distributions of $\mathrm{D}^{0}$ and $\mathrm{D}^{+}$candidates and distributions of the mass difference between the $\mathrm{D}^{0} \pi^{+}$and the $\mathrm{D}^{0}$ candidate, in the case of channels involving a $\mathrm{D}^{*+}$, have been used to define signal and sidebands samples (Figure 1). Events from the sidebands have been used to evaluate the level of the combinatorial background under the $\mathrm{D}$ or $\mathrm{D}^{*+}$ signal.

For $\mathrm{D}^{* *}$ states, decay channels into a $\mathrm{D}^{(*)}$ and, at most, two pions have been considered. When searching for $\mathrm{D}^{* *} \rightarrow \mathrm{D}^{0} \pi^{+}$decays, a veto has been applied against the $\mathrm{D}^{*+}$ by removing candidates with a mass difference $m\left(\mathrm{D}^{0} \pi^{+}\right)-m\left(\mathrm{D}^{0}\right)<0.1465 \mathrm{GeV} / c^{2}$, for $\mathrm{D}^{0}$ mesons decaying into charged particles only ${ }^{3}$. As the combinatorial background under $\mathrm{D}$ signals is higher than for channels involving a $\mathrm{D}^{*+}$, values of the cuts given in [29] have been made tighter for $\mathrm{D}^{* *} \rightarrow \mathrm{D} \pi$ events, as compared with those applied to $\mathrm{D}^{* *} \rightarrow \mathrm{D}^{*+} \pi$ decays.

Events have been selected by reconstructing a lepton, a $\mathrm{D}^{(*)}$ and a charged pion whose trajectories are compatible with the hypothesis that they originate from a common secondary vertex. $\mathrm{D}^{* *}$ decays considered in the present analysis involve always at least one

\footnotetext{
${ }^{3}$ For $\mathrm{D}^{0} \pi$ final states, only $\mathrm{D}^{0}$ decaying into $\mathrm{K}^{-} \pi^{+}$or $\mathrm{K}^{-} \pi^{+} \pi^{+} \pi^{-}$have been used.
} 

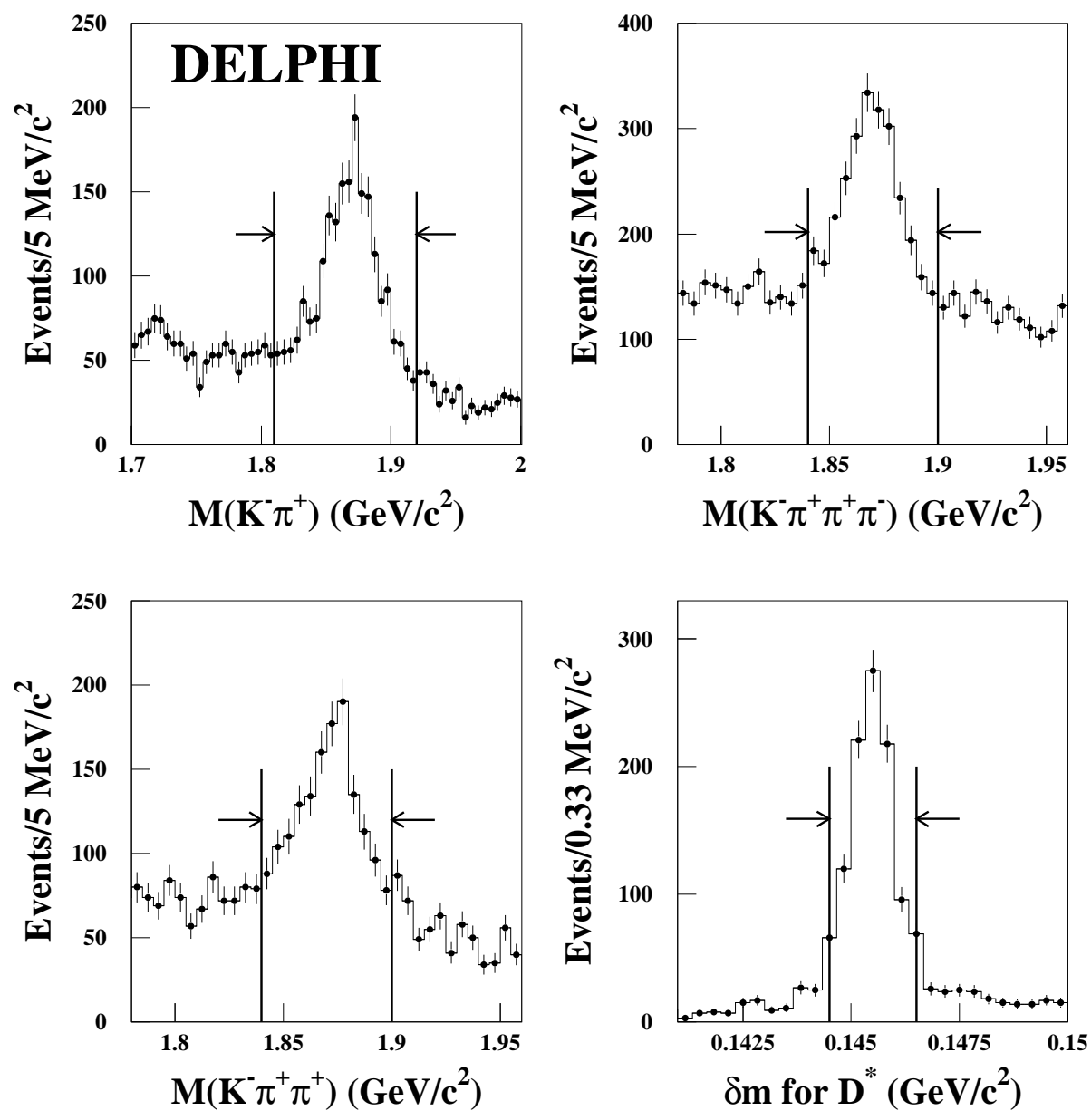

Figure 1: $\mathrm{D}^{0}, \mathrm{D}^{+}$and $\mathrm{D}^{*+}$ signals used in the present analysis, in which the $\mathrm{D}^{0}$ meson decays into charged particles only and which correspond to events registered in 1992-95. Intervals used to define the signal and sideband regions are indicated. 
charged particle track ${ }^{4}$ emitted at the $b$-decay vertex, in addition to the exclusively reconstructed $\mathrm{D}^{(*)}$ meson. The $\pi^{* *}$ momentum has been required to be higher than $0.5 \mathrm{GeV} / c$ and the track must be associated to at least one measurement in the Vertex Detector.

The overall efficiencies for selecting signal events (see Table 3), not including the decay branching fractions of $\mathrm{D}^{*+}, \mathrm{D}^{0}$ or $\mathrm{D}^{+}$mesons into their considered decay channels, have been estimated from simulated events. Efficiencies are rather similar for the 92-93 and 94-95 samples when considering channels with a $\mathrm{D}^{*+}$ or with $\mathrm{D}^{0} \rightarrow \mathrm{K}^{-} \pi^{+}$in spite of the reduced performances of the VD for the 92-93 period. This is because, as the background level is rather low for these channels, loose cuts on the vertex separation have been applied. For the other channels, which require tighter cuts, efficiencies are markedly lower in 92-93. These values have already been corrected for differences between the actual measured lifetimes of $b$-hadrons and that used in the simulation.

\begin{tabular}{|c|c|c|}
\hline decay channel & $92-93 \mathrm{MC}$ & $94-95 \mathrm{MC}$ \\
\hline \hline $\mathrm{D}^{* *} \rightarrow \mathrm{D}^{0} \pi^{+}$ & & \\
\hline $\mathrm{D}^{0} \rightarrow \mathrm{K}^{-} \pi^{+}$ & $(10.4 \pm 0.5) \%$ & $(13.0 \pm 0.5) \%$ \\
$\mathrm{D}^{0} \rightarrow \mathrm{K}^{-} \pi^{+} \pi^{+} \pi^{-}$ & $(3.0 \pm 0.3) \%$ & $(4.8 \pm 0.3) \%$ \\
\hline \hline $\mathrm{D}^{* *} \rightarrow \mathrm{D}^{+} \pi^{-}$ & & \\
\hline $\mathrm{D}^{+} \rightarrow \mathrm{K}^{-} \pi^{+} \pi^{+}$ & $(5.9 \pm 0.3) \%$ & $(9.1 \pm 0.3) \%$ \\
\hline \hline $\mathrm{D}^{* *} \rightarrow \mathrm{D}^{*+} \pi^{-}$ & & \\
\hline $\mathrm{D}^{0} \rightarrow \mathrm{K}^{-} \pi^{+}$ & $(12.0 \pm 0.3) \%$ & $(13.9 \pm 0.4) \%$ \\
$\mathrm{D}^{0} \rightarrow \mathrm{K}^{-} \pi^{+} \pi^{+} \pi^{-}$ & $(5.0 \pm 0.2) \%$ & $(5.8 \pm 0.2) \%$ \\
$\mathrm{D}^{0} \rightarrow \mathrm{K}^{-} \pi^{+}\left(\pi^{0}\right)$ & $(6.6 \pm 0.6) \%$ & $(7.3 \pm 0.6) \%$ \\
\hline
\end{tabular}

Table 3: Global efficiencies of the analysis chain to reconstruct and select simulated signal events. Quoted uncertainties are only statistical. In addition to the simulated events mentioned in Table 1, dedicated event samples corresponding to the different channels have been used to increase the statistics.

\subsection{Signal separation from background sources}

When considering $\mathrm{D}^{(*)} \pi^{ \pm}$combinations, the main sources of background can be divided into two categories depending on whether they correspond or not to a real reconstructed $\mathrm{D}^{(*)}$ meson. The latter is the combinatorial background situated under the charm mass signal. Background events with a real $\mathrm{D}^{(*)}$ can originate from the following sources:

- the $\pi^{* *}$ candidate is not produced at the $b$-decay vertex but comes from the beam interaction point (primary pion background);

- the lepton originates from the weak decay of another charm particle emitted in the b-decay (cascade background);

- the lepton originates from a $\tau$ decay (tau background);

- the candidate lepton is a misidentified hadron or a converted photon (fake lepton background);

- the reconstructed charm meson originates from a $c \bar{c}$ event (charm background).

A variable, used to isolate the signal from these backgrounds, has been defined from the probability distributions of several discriminant observables, whose shapes have been

\footnotetext{
${ }^{4}$ This particle is called $\pi^{* *}$ in the following analysis.
} 
obtained from the simulation and also directly from data, as in the case of the combinatorial background. The following observables have been used:

- the lifetime-signed impact parameters of the $\pi^{* *}$ relative to the main vertex of the event, in $\mathrm{R} \phi$ and $z$ projections, normalised to their uncertainty;

- the normalised and lifetime-signed impact parameters of the $\pi^{* *}$ relative to the secondary vertex, in $\mathrm{R} \phi$ and $z$ projections;

- the normalised and lifetime-signed decay distance between the primary and the secondary vertices;

- the cosine of the decay angle defined as the angle of the $\pi^{* *}$ direction, boosted to the $b$-hadron rest frame, relative to the $\mathrm{B}$ direction. This variable is uniformly distributed for the signal whereas it is peaked at negative values for backgrounds;

- the $\chi^{2}$ probability for the secondary vertex, which should be uniformly distributed for the signal and peaked at small values for the main sources of background;

- the two variables, $d_{ \pm}$, which depend on the presence of additional charged particles at the secondary vertex. They are defined in the following way:

- all charged particles, other than the $\mathrm{D}^{* *}$ decay products and the lepton, emitted in the same event hemisphere as the $b$-candidate, with momentum larger than $500 \mathrm{MeV} / c$, which form a mass with the $\mathrm{D}^{(*)} \pi^{* *} \ell^{-}$system lower than $6 \mathrm{GeV} / c^{2}$ and which have values for their impact parameters relative to the $b$-decay vertex smaller than 2 and $1.5 \sigma$ in $\mathrm{R} \phi$ and $z$ respectively, are considered;

- selected particles, having the same $(+)$ or the opposite $(-)$ charge as the lepton are considered separately. If there are several candidates in a class, the one with the largest impact parameter to the main vertex is retained and the quantity:

$$
x_{ \pm}=\epsilon(\mathrm{R} \phi) \times n \operatorname{sig}(\mathrm{R} \phi)^{2}+\epsilon(z) \times n \operatorname{sig}(z)^{2}
$$

is evaluated, where $\epsilon$ and $n s i g$ are, respectively, the sign and the number of standard deviations of the track impact parameter relative to the main vertex ${ }^{5}$. The sign of the impact parameter is taken to be positive (negative) if the corresponding track trajectory intercepts the line of the jet axis from the main vertex downstream (upstream) from that vertex.

As the track impact parameters can extend to very large values because of the relatively long decay time of $b$-hadrons, the variables $d_{ \pm}$are taken to be equal to the logarithm of $\left(1+x_{ \pm}^{2}\right)$ and their sign is taken to be the same as $x_{ \pm}$. For events with no spectator track candidate, that is, with no additional tracks compatible with the $b$-decay vertex, a fixed value of -4.0 is used for $d_{ \pm}$. For $\mathrm{D}^{(*)} \pi$ signal events, it is expected that no additional track is present at the $b$-decay vertex whereas for $\mathrm{D}^{(*)} \pi^{+} \pi^{-}$candidates, another track with a precisely defined charge correlation with the lepton, is expected. These properties have been used in the analysis in the following way. Selected events have been distributed into right-sign and wrong-sign candidates. Right-sign events correspond to $\mathrm{D}^{0} \pi^{+}, \mathrm{D}^{+} \pi^{-}$and $\mathrm{D}^{*+} \pi^{-}$pairs whereas wrong-sign events have an opposite sign pion. Since only $\mathrm{D}^{* *+}$ or $\mathrm{D}^{* * 0}$ states can be produced in the semileptonic decay of a $b$-hadron, where they are accompanied by a negatively charged lepton, wrong-sign combinations can receive contributions only from $\mathrm{D}^{(*)} \pi^{+} \pi^{-}$final states. For right-sign combinations it has been required that signal events behave as if there is no additional charged particle track at the $b$-decay vertex. This implies that, in the case of $\mathrm{D}^{(*)} \pi \pi$ decays, only $\mathrm{D}^{0} \pi^{+} \pi^{0}$ decays or those involving two charged pions and where the $\pi^{-}$escapes detection, can contribute. For

\footnotetext{
${ }^{5}$ The second term of this equation is not considered for 1992-1993 data since the $z$ coordinate is not measured.
} 
wrong-sign combinations, it has been required that signal events behave as if there was another charged particle track, of sign opposite to the $\pi^{* *}$ and of trajectory compatible with the $b$-decay vertex position.

Probability distributions for each of these nine (seven for 1992-1993 data as the vertex detector measured only $\mathrm{R} \phi$ coordinates) variables, have been obtained using the simulation for signal and background events. The agreement in the shape of the distributions of these variables for real and simulated events is illustrated in Figure 2-left in which the $\chi^{2}$ probability distributions for secondary vertices for events selected in the sidebands of the signal have been compared. This distribution was selected as it is sensitive to possible differences between real and simulated events.

The probabilities $P_{\text {signal }}$ and $P_{b a c k g}$. have been obtained by multiplying the probability of each discriminating variable and a global discriminant $R$ has been defined as:

$$
R=\frac{P_{\text {signal }}-P_{\text {backg. }}}{P_{\text {signal }}+P_{\text {backg. }}},
$$

in which events corresponding to the two largest background sources, namely the combinatorial and the primary pion background components, have been used to determine the probabilities for background events. The distribution of the variable $R$ is peaked at $+1(-1)$ for signal (background) events (see Figure 3).

In Figure 2-right the distributions of this quantity for combinatorial background events selected in the sidebands of the $\mathrm{D}^{0}$ signal for real and simulated events are compared. These distributions are in agreement but, to account for possible differences between real and simulated events and as the combinatorial background component is the largest of the background components for $\mathrm{D}^{0} \pi$ and $\mathrm{D}^{+} \pi$ final states, real events situated in the sidebands of the signal have been used, in the following, to determine also the shape of the $R$ distribution for the combinatorial background component.

\section{3 $\quad \mathrm{D}^{* *}$ production rates}

Events corresponding to the $\mathrm{D}^{0} \rightarrow \mathrm{K}^{-} \pi^{+}\left(\pi^{0}\right)$ decay channel have not been included in this study, as they are affected by a larger combinatorial background.

The $R$ variable distributions, for the different $\mathrm{D}^{(*)}$ decay channels, have been measured separately for right- and wrong-sign samples and for the two data-taking periods. A binned likelihood fit [30] has been performed ${ }^{6}$ in order to measure the branching fraction in each channel. The fractions of signal and primary pion background events have been left free to vary in the fit whereas the fractions of the other components with a real $\mathrm{D}^{(*)}$ have been fixed to values taken from external measurements. The discriminant variable distribution for combinatorial background events has been obtained from data using the sideband events. Distributions for the other background components have been taken from the simulation.

Fractions which have been kept fixed in the fit have then been varied, in turn, according to their expected overall accuracy and the fit repeated to estimate the corresponding systematic errors. The fitting procedure has been verified on simulated events (see the last column of Tables 5-6).

The simulation of cascade events comprises $b \rightarrow \mathrm{D}_{s}^{-} \mathrm{D} X$ events only. Corrections have therefore been applied to the rate and the topology of these events to account for the missing decay modes. Present measurements from ALEPH [31] and BaBar [32] on double

${ }^{6}$ Statistical uncertainties due to the finite number of analyzed simulated events are included in this fit. 

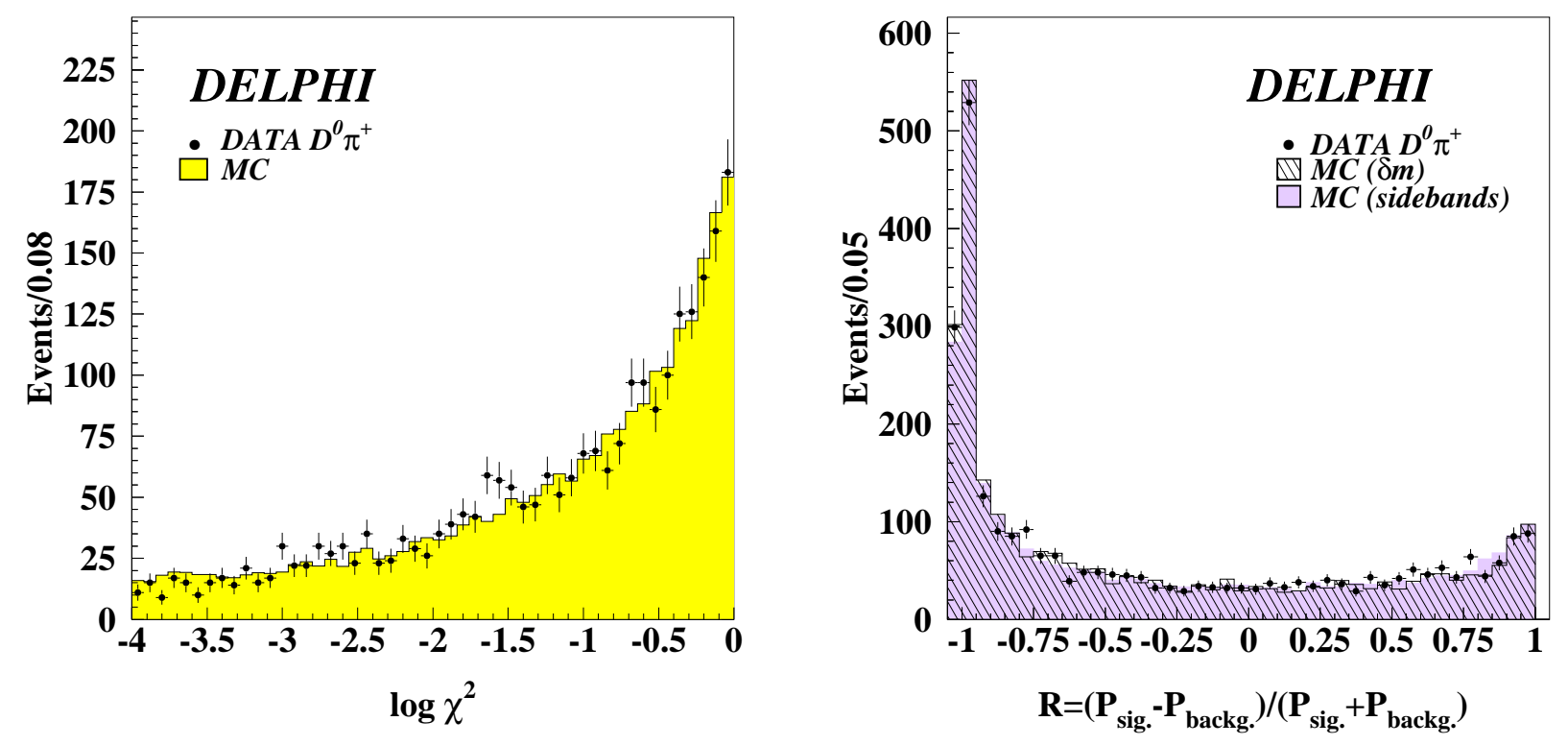

Figure 2: Left: Distributions of the decimal logarithm of the $\chi^{2}$ probability for secondary $\mathrm{D}^{0} \pi \ell^{-}$vertices in events selected in the sidebands of the signal obtained using real (points with error bars) and simulated (histogram) events. Right: Distributions of the values of the discriminant variable corresponding to events selected in the sidebands of the signal for real data (points with error bars) and simulated events (shadowed histogram). The other histogram (hatched) corresponds to simulated events from combinatorial background situated in the signal mass region. The histograms have been normalized to the same number of entries. 



Figure 3: Discriminant variable distributions for a) $\mathrm{D}^{*+} \pi^{-}$and b) $\mathrm{D}^{*+} \pi^{+}$candidates. Points with error bars correspond to real events whereas the histograms show the fitted components. The fake lepton background in these plot includes $\tau$ events.

charm decays of $b$-hadrons have been used, together with results on $c$-hadron inclusive and exclusive semileptonic decays from [21], to determine these corrections. They depend on the topology of the studied channel. For each channel, the correction factor on the branching fraction and the probabilities to have no track of same $\left(\mathcal{P}(0)_{s s}\right)$ or opposite-sign $\left(\mathcal{P}(0)_{\text {os }}\right)$ as the lepton have been evaluated (see Table 4$)$.

\begin{tabular}{|c|c|c|c|c|}
\hline Channel & Expected rate $(\%)$ & corr. factor & $\mathcal{P}(0)_{\text {ss }}(\%)$ & $\mathcal{P}(0)_{\text {os }}(\%)$ \\
\hline Right sign candidates & & & & \\
\hline$b \rightarrow \mathrm{D}^{0} \pi^{+} \ell^{-} X$, veto on $\mathrm{D}^{*+}$ & $1.61 \pm 0.29$ & 3.5 & $33 \pm 4$ & $33 \pm 4$ \\
$b \rightarrow \mathrm{D}^{+} \pi^{-} \ell^{-} X$ & $0.54 \pm 0.13$ & 2.3 & $63 \pm 4$ & $15 \pm 3$ \\
$b \rightarrow \mathrm{D}^{*+} \pi^{-} \ell^{-} X$ & $0.65 \pm 0.26$ & 2.7 & $69 \pm 5$ & $13 \pm 3$ \\
\hline Wrong sign candidates & & & & \\
\hline$b \rightarrow \mathrm{D}^{0} \pi^{-} \ell^{-} X$, veto on $\mathrm{D}^{*+}$ & $0.58 \pm 0.12$ & 2.6 & $\sim 1$ & $\sim 0$ \\
$b \rightarrow \mathrm{D}^{+} \pi^{+} \ell^{-} X$ & $0.43 \pm 0.09$ & 3.4 & $13 \pm 2$ & $90 \pm 3$ \\
$b \rightarrow \mathrm{D}^{*+} \pi^{+} \ell^{-} X$ & $0.58 \pm 0.17$ & 4.2 & $22 \pm 3$ & $89 \pm 2$ \\
\hline
\end{tabular}

Table 4: Double-charm expected rates and probabilities for having no additional charged track in the considered decay channels.

Measured branching fractions are summarized in Tables 5 and 6 respectively for rightand wrong-sign candidates.

The following contributions to the systematic uncertainties have been considered:

- uncertainties related to the values of external parameters, such as $R_{b}$, and the different branching fractions of charmed hadrons into the reconstructed final states. The values used in the present analysis, taken from [21], are given in Table 7; 


\begin{tabular}{|c|c|c|c|}
\hline $\begin{array}{c}\text { Right sign candidates } \\
\text { Channel }\end{array}$ & $\begin{array}{c}\text { number of } \\
\text { fitted events }\end{array}$ & $\begin{array}{l}\text { branching } \\
\text { fraction }(\%)\end{array}$ & $\begin{array}{c}\text { number of events } \\
\text { fitted (expected) MC }\end{array}$ \\
\hline \multicolumn{4}{|l|}{ 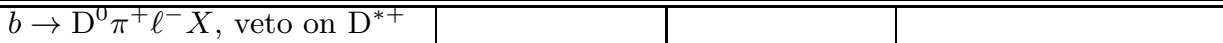 } \\
\hline $\mathrm{D}^{0} \rightarrow \mathrm{K}^{-} \pi^{+}(92-93)$ & $109.0 \pm 27.5$ & $2.34 \pm 0.59$ & $515.2 \pm 67.7(435)$ \\
\hline $\mathrm{D}^{0} \rightarrow \mathrm{K}^{-} \pi^{+}(94-95)$ & $60.3 \pm 20.3$ & $0.69 \pm 0.23$ & $939.6 \pm 67.5(1001)$ \\
\hline $\mathrm{D}^{0} \rightarrow \mathrm{K}^{-} \pi^{+} \pi^{+} \pi^{-}(92-93)$ & $13.2 \pm 20.0$ & $0.51 \pm 0.77$ & $262.8 \pm 50.9(243)$ \\
\hline $\mathrm{D}^{0} \rightarrow \mathrm{K}^{-} \pi^{+} \pi^{+} \pi^{-}(94-95)$ & $59.0 \pm 20.7$ & $0.93 \pm 0.33$ & $774.5 \pm 64.5(756)$ \\
\hline Average & & $0.89 \pm 0.18$ & \\
\hline \multicolumn{4}{|l|}{ 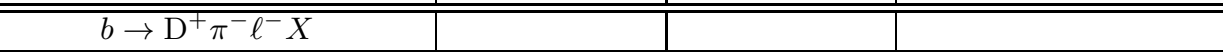 } \\
\hline $\mathrm{D}^{+} \rightarrow \mathrm{K}^{-} \pi^{+} \pi^{+}(92-93)$ & $47.4 \pm 18.8$ & $0.76 \pm 0.30$ & $350.1 \pm 47.6(394)$ \\
\hline $\mathrm{D}^{+} \rightarrow \mathrm{K}^{-} \pi^{+} \pi^{+}$(94-95) & $36.2 \pm 16.5$ & $0.25 \pm 0.11$ & $1101.1 \pm 63.0(1132)$ \\
\hline Average & & $0.31 \pm 0.10$ & \\
\hline \multicolumn{4}{|l|}{$\bar{b} \rightarrow \mathrm{D}^{*+} \pi^{-} \ell^{-} X$} \\
\hline $\mathrm{D}^{0} \rightarrow \mathrm{K}^{-} \pi^{+}(92-93)$ & $13.2 \pm 9.3$ & $0.36 \pm 0.26$ & $125.3 \pm 23.6(128)$ \\
\hline $\mathrm{D}^{0} \rightarrow \mathrm{K}^{-} \pi^{+}(94-95)$ & $32.8 \pm 8.8$ & $0.52 \pm 0.14$ & $282.6 \pm 28.7(303)$ \\
\hline $\mathrm{D}^{0} \rightarrow \mathrm{K}^{-} \pi^{+} \pi^{+} \pi^{-}(92-93)$ & $16.4 \pm 7.1$ & $0.55 \pm 0.24$ & $111.2 \pm 20.2(96)$ \\
\hline $\mathrm{D}^{0} \rightarrow \mathrm{K}^{-} \pi^{+} \pi^{+} \pi^{-}(94-95)$ & $12.9 \pm 7.2$ & $0.25 \pm 0.14$ & $239.5 \pm 28.0(227)$ \\
\hline Average & & $0.40 \pm 0.09$ & \\
\hline
\end{tabular}

Table 5: Measured branching fractions for $b \rightarrow \mathrm{D} \pi \ell^{-} X$ in right-sign combinations using the $\mathrm{D}$ decay branching fractions taken from Table 7 . The last column gives the results obtained on simulated events. Numbers within parentheses correspond to the real number of simulated signal.

- detector-dependent uncertainties such as those on the tracking efficiency, on the lepton identification efficiency and on the correction of differences between real and simulated events relative to the fake lepton rate;

- differences between real and simulated events on track reconstruction accuracy. Distributions of the discriminant variable for signal-like events, obtained in real and simulated events, have also been compared using $\mathrm{D}^{*+} \rightarrow \mathrm{D}^{0} \pi^{+}$decays, with $\mathrm{D}^{0} \rightarrow \mathrm{K}^{-} \pi^{+}$or $\mathrm{K}^{-} \pi^{+} \pi^{+} \pi^{-}$. These events have been analysed using the same criteria as those applied to $\mathrm{D}^{* *} \rightarrow \mathrm{D}^{0} \pi^{+}$candidates. A discriminant variable $\left(R^{*}\right)$ is constructed using the same input quantities as for the $R$ variable, apart from $d_{ \pm}$ whose effects have been evaluated separately. Possible differences between real and simulated events affecting track offset measurements, decay length reconstruction and $\chi^{2}$ probability of secondary vertices, in the case of a signal, can then be studied. Distributions of the $R^{*}$ variable obtained using 461 reconstructed $\mathrm{D}^{*+}$ in real data and 4100 in simulated events are compared in Figure 4. The two distributions agree within the statistical uncertainties. A possible difference in the shape of these distributions has been parametrized assuming a linear variation with the value of $R^{*}$. The fitted slope is equal to $-0.01 \pm 0.08$. In the following, the effect of a variation on the shape of the discriminant variable distribution, induced by a linear correction of slope equal to \pm 0.1 , has been evaluated;

- corrections of the cascade decay rate applied to simulated events have been evaluated with a $30 \%$ relative uncertainty (see Table 4). This variation is larger than most of the uncertainties attached to the correction applied to each channel and covers the dispersion of the values of these corrections. Variations in the cascade rates have been applied in a correlated manner to all channels when this was relevant. Effects from possible differences between real and simulated events for the $d_{ \pm}$distributions have been evaluated in [29] by studying the decay channel $b \rightarrow \mathrm{D}^{*+} \mathrm{X} \ell^{-} \bar{\nu}_{\ell}$. The probability to have no additional track, for signal events in which there is in reality 


\begin{tabular}{|c|c|c|c|}
\hline $\begin{array}{c}\text { Wrong sign candidates } \\
\text { Channel }\end{array}$ & $\begin{array}{c}\text { number of } \\
\text { fitted events }\end{array}$ & $\begin{array}{c}\text { branching } \\
\text { fraction }(\%)\end{array}$ & $\begin{array}{c}\text { number of events } \\
\text { fitted (expected) MC }\end{array}$ \\
\hline \multicolumn{4}{|l|}{ 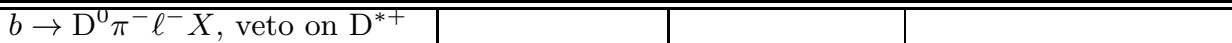 } \\
\hline $\mathrm{D}^{0} \rightarrow \mathrm{K}^{-} \pi^{+}(92-93)$ & $34.3 \pm 17.8$ & $0.74 \pm 0.38$ & $57.0 \pm 41.9(70)$ \\
\hline $\mathrm{D}^{0} \rightarrow \mathrm{K}^{-} \pi^{+}(94-95)$ & $26.3 \pm 15.3$ & $0.30 \pm 0.17$ & $136.8 \pm 36.6(141)$ \\
\hline $\mathrm{D}^{0} \rightarrow \mathrm{K}^{-} \pi^{+} \pi^{+} \pi^{-}(92-93)$ & $3.5 \pm 14.1$ & $0.14 \pm 0.56$ & $25.1 \pm 33.1(40)$ \\
\hline $\mathrm{D}^{0} \rightarrow \mathrm{K}^{-} \pi^{+} \pi^{+} \pi^{-}(94-95)$ & $1.4 \pm 15.5$ & $0.02 \pm 0.23$ & $143.1 \pm 41.1(166)$ \\
\hline Average & & $0.26 \pm 0.13$ & \\
\hline \multicolumn{4}{|l|}{$\overline{c b \rightarrow \mathrm{D}^{+} \pi^{+} \ell^{-} X}$} \\
\hline $\mathrm{D}^{+} \rightarrow \mathrm{K}^{-} \pi^{+} \pi^{+}(92-93)$ & $10.1 \pm 17.2$ & $+0.16 \pm 0.27$ & $119.8 \pm 20.0(87)$ \\
\hline $\mathrm{D}^{+} \rightarrow \mathrm{K}^{-} \pi^{+} \pi^{+}$(94-95) & $-9.9 \pm 15.8$ & $-0.07 \pm 0.11$ & $190.6 \pm 44.2(226)$ \\
\hline Average & & $-0.04 \pm 0.10$ & \\
\hline \multicolumn{4}{|l|}{$\overline{b \rightarrow \mathrm{D}^{*+}} \pi^{+} \ell^{-} X$} \\
\hline $\mathrm{D}^{0} \rightarrow \mathrm{K}^{-} \pi^{+}(92-93)$ & $-0.3 \pm 8.3$ & $-0.01 \pm 0.22$ & not simul. \\
\hline $\mathrm{D}^{0} \rightarrow \mathrm{K}^{-} \pi^{+}(94-95)$ & $0.62 \pm 6.3$ & $+0.01 \pm 0.10$ & - \\
\hline $\mathrm{D}^{0} \rightarrow \mathrm{K}^{-} \pi^{+} \pi^{+} \pi^{-}(92-93)$ & $9.0 \pm 6.5$ & $+0.30 \pm 0.22$ & - \\
\hline $\mathrm{D}^{0} \rightarrow \mathrm{K}^{-} \pi^{+} \pi^{+} \pi^{-}(94-95)$ & $-8.2 \pm 7.8$ & $-0.16 \pm 0.15$ & - \\
\hline Average & & $0.00 \pm 0.07$ & \\
\hline
\end{tabular}

Table 6: Measured branching fractions for the different wrong-sign combinations. The last column gives the results obtained on simulated events and numbers within parentheses correspond to the real simulated signal.

no other track coming from the $b$-vertex, is of the order of $80 \%$ and we have taken a $5 \%$ relative error. For events, in which there is at least one such track produced at the secondary vertex, the probability to miss it is estimated to be $(20 \pm 10 \%)$. For events selected in the $\delta m$ region, situated above the $\mathrm{D}^{*+}$ signal, in real and simulated events, the probabilities for having no spectator track are of the order of $34 \%$ and differ by $(2.5 \pm 1.5) \%$.

The uncertainty related to the finite statistics of simulated events has been included in the statistical uncertainty of fitted signal event numbers.

\begin{tabular}{|c|c|}
\hline $\begin{array}{c}\text { parameter } \\
\text { or hypothesis }\end{array}$ & $\begin{array}{c}\text { central value } \\
\text { and uncertainty }\end{array}$ \\
\hline $\mathrm{R}_{b}$ & $0.21664 \pm 0.00068$ \\
$\mathrm{BR}\left(\mathrm{D}^{*+} \rightarrow \mathrm{D}^{0} \pi^{+}\right)$ & $0.677 \pm 0.005$ \\
$\mathrm{BR}\left(\mathrm{D}^{0} \rightarrow \mathrm{K}^{-} \pi^{+}\right)$ & $0.0380 \pm 0.0009$ \\
$\mathrm{BR}\left(\mathrm{D}^{0} \rightarrow \mathrm{K}^{-} \pi^{+} \pi^{+} \pi^{-}\right)$ & $0.0746 \pm 0.0031$ \\
$\mathrm{BR}\left(\mathrm{D}^{0} \rightarrow \mathrm{K}^{-} \pi^{+} \pi^{0}\right)$ & $0.131 \pm 0.009$ \\
$\mathrm{BR}\left(\mathrm{D}^{0} \rightarrow \mathrm{K}^{-} \ell^{+} \nu_{\ell}\right)$ & $0.070 \pm 0.003$ \\
$\mathrm{BR}\left(\mathrm{D}^{0} \rightarrow \mathrm{K}^{-} \mathrm{K}^{+}\right)$ & $0.00412 \pm 0.00014$ \\
$\mathrm{BR}\left(\mathrm{D}^{+} \rightarrow \mathrm{K}^{-} \pi^{+} \pi^{+}\right)$ & $0.091 \pm 0.006$ \\
$\mathrm{P}\left(b \rightarrow \overline{\mathrm{B}_{\mathrm{d}}^{0}}\right)$ & $0.388 \pm 0.013$ \\
$\mathrm{P}\left(b \rightarrow \overline{\mathrm{B}_{\mathrm{s}}^{0}}\right)$ & $0.106 \pm 0.013$ \\
$\tau\left(\overline{\mathrm{B}_{\mathrm{d}}^{0}}\right)$ & $1.542 \pm 0.016 \mathrm{ps}$ \\
\hline
\end{tabular}

Table 7: Values for the external parameters used in the analysis [21].

A summary of these contributions is given in Table 8 . 


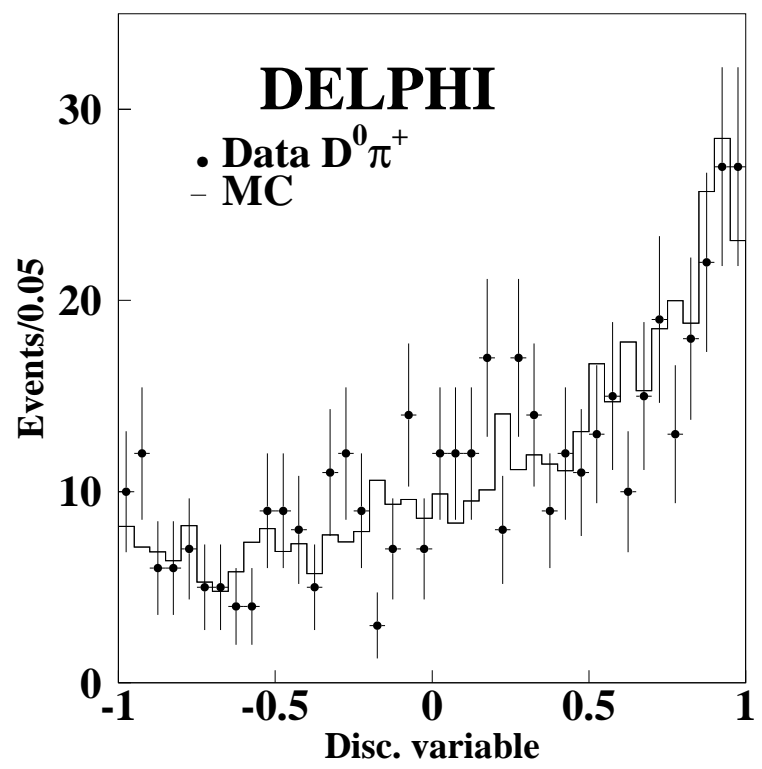

Figure 4: Discriminant variable distributions for $\mathrm{D}^{*+} \rightarrow \mathrm{D}^{0} \pi^{+}$candidates selected in data (points with error bars) and in simulated hadronic $\mathrm{Z}$ decays (histogram).

Measured branching fractions, for right-sign events, are summarized in Table 9, where they are compared with similar results obtained in other experiments. The present analysis supersedes the previous DELPHI analysis [25], in terms of statistical accuracy, as more information has been used to separate signal and background events, and systematics. All experimental results are compatible.

There is no significant excess of events in wrong-sign combinations. Measurements of the $\mathrm{D}^{0} \pi^{-}$and $\mathrm{D}^{+} \pi^{+}$channels can be averaged, independently of the isospin of the $\pi \pi$ system, as the same number of events is expected in the two channels giving:

$$
\begin{gathered}
\mathrm{BR}\left(b \rightarrow \mathrm{D}^{0} \pi^{+} \pi^{-} \ell^{-} \bar{\nu}_{\ell}\right)=\mathrm{BR}\left(b \rightarrow \mathrm{D}^{+} \pi^{+} \pi^{-} \ell^{-} \bar{\nu}_{\ell}\right)=(0.06 \pm 0.08 \pm 0.04) \% \\
\operatorname{BR}\left(b \rightarrow \mathrm{D}^{*+} \pi^{+} \pi^{-} \ell^{-} \bar{\nu}_{\ell}\right)=(0.00 \pm 0.07 \pm 0.07) \%
\end{gathered}
$$

The values corresponding to $90 \%$ C.L. upper limits are equal to, respectively, $0.18 \%$ and

\begin{tabular}{|c|c|c|c|c|c|c|c|}
\hline $\begin{array}{l}\text { Right sign } \\
\text { candidates }\end{array}$ & $\begin{array}{c}\text { External } \\
\mathrm{BR}, R_{b}\end{array}$ & $\begin{array}{l}\text { Tracking } \\
\text { efficiency }\end{array}$ & $\begin{array}{l}\text { Lepton ID } \\
\text { efficiency }\end{array}$ & $\begin{array}{l}\text { Fake lept. } \\
\text { rate }\end{array}$ & $\begin{array}{l}\text { Discr. } \\
\text { var. }\end{array}$ & $\begin{array}{c}\text { Cascade } \\
\text { modelling }\end{array}$ & $\overline{\text { Total }}$ \\
\hline$b \rightarrow \mathrm{D}^{0} \pi^{+} \ell^{-} X$ & 0.028 & 0.012 & 0.016 & 0.006 & 0.047 & 0.033 & 0.067 \\
\hline$b \rightarrow \mathrm{D}^{+} \pi^{-} \ell^{-} X$ & 0.021 & 0.005 & 0.006 & 0.003 & 0.007 & 0.011 & 0.026 \\
\hline$b \rightarrow \mathrm{D}^{*+} \pi^{-} \ell^{-} X$ & 0.014 & 0.007 & 0.007 & 0.003 & 0.015 & 0.013 & 0.027 \\
\hline $\begin{array}{l}\text { Wrong sign } \\
\text { candidates }\end{array}$ & & & & & & & \\
\hline$b \rightarrow \mathrm{D}^{0} \pi^{-} \ell^{-} X$ & 0.028 & 0.012 & 0.016 & 0.006 & 0.047 & 0.053 & 0.079 \\
\hline$b \rightarrow \mathrm{D}^{+} \pi^{+} \ell^{-} X$ & 0.021 & 0.005 & 0.006 & 0.002 & 0.007 & 0.017 & 0.029 \\
\hline$b \rightarrow \mathrm{D}^{*+} \pi^{+} \ell^{-} X$ & 0.014 & 0.007 & 0.007 & 0.004 & 0.015 & 0.067 & 0.071 \\
\hline
\end{tabular}
$0.13 \%$.

Table 8: Contributions of systematic uncertainties (in $10^{-2}$ units) to the measured production rates of $b \rightarrow \mathrm{D}^{(*)} \pi X \ell^{-} \bar{\nu}_{\ell}$ events. 


\begin{tabular}{|c|c|c|c|}
\hline $\begin{array}{c}\text { Right sign } \\
\text { candidates }\end{array}$ & $\begin{array}{c}\text { DELPHI } \\
\text { this analysis }\end{array}$ & $\begin{array}{c}\text { DELPHI } \\
{[25]}\end{array}$ & $\begin{array}{c}\text { ALEPH } \\
{[19]}\end{array}$ \\
\hline$b \rightarrow \mathrm{D}^{0} \pi^{+} \ell^{-} X$ & $8.9 \pm 1.8 \pm 0.7$ & $11.6 \pm 2.4 \pm 1.1$ & $7.3 \pm 1.8 \pm 1.0$ \\
$b \rightarrow \mathrm{D}^{+} \pi^{-} \ell^{-} X$ & $3.1 \pm 1.0 \pm 0.3$ & $4.9 \pm 1.8 \pm 0.7$ & $3.0 \pm 0.7 \pm 0.5$ \\
$b \rightarrow \mathrm{D}^{*+} \pi^{-} \ell^{-} X$ & $4.0 \pm 0.9 \pm 0.3$ & $4.8 \pm 0.9 \pm 0.5$ & $4.7 \pm 0.8 \pm 0.6$ \\
\hline Wrong sign & & & \\
candidates & & & \\
\hline$b \rightarrow \mathrm{D}^{0} \pi^{-} \ell^{-} X$ & $2.6 \pm 1.3 \pm 0.8$ & $2.3 \pm 1.5 \pm 0.4$ & \\
$b \rightarrow \mathrm{D}^{+} \pi^{+} \ell^{-} X$ & $-0.4 \pm 1.0 \pm 0.3$ & $2.6 \pm 1.5 \pm 0.4$ & \\
$b \rightarrow \mathrm{D}^{*+} \pi^{+} \ell^{-} X$ & $0.0 \pm 0.7 \pm 0.7$ & $0.6 \pm 0.7 \pm 0.2$ & \\
\hline
\end{tabular}

Table 9: Comparison between measured production rates (in $10^{-3}$ units) of $b \rightarrow \mathrm{D}^{(*)} \pi X \ell^{-} \bar{\nu}_{\ell}$ events.

\subsection{Study of the $\mathrm{D}^{* *}$ hadronic mass distribution}

In order to study the mass distribution of right sign events, corresponding to the $\mathrm{D}^{* *}$ signal, the cut $R>0.25$ has been applied on the discriminant variable to reduce the contribution from background events. This cut corresponds to a selection efficiency which varies between $67 \%$ and $85 \%$ for signal events, depending on the channel and on the data sample.

A maximum likelihood fit has been performed using the $\Delta_{m}=m\left(\mathrm{D}^{(*)} \pi^{* *}\right)-m\left(\mathrm{D}^{(*)}\right)$ and the $R$ variables, introducing the following components and parameters:

- $b_{D_{0}^{*}}=\mathrm{BR}\left(\overline{\mathrm{B}_{\mathrm{d}}^{0}} \rightarrow \mathrm{D}_{0}^{*+} \ell^{-} \bar{\nu}_{\ell}\right)$. In practice this component is considered to be a broad resonant mass distribution which can account for various possible states;

- $m_{D_{0}^{*}}$ : the mass of the $\mathrm{D}_{0}^{*}$ : it is kept fixed at $2.4 \mathrm{GeV} / c^{2}$ and a scan of its possible values between 2.3 and $2.5 \mathrm{GeV} / c^{2}$ has been made;

- $\Gamma_{D_{0}^{*}}$ : the total width of the $\mathrm{D}_{0}^{*}$;

- $b_{D_{1}^{*}}=\operatorname{BR}\left(\overline{\mathrm{B}_{\mathrm{d}}^{0}} \rightarrow \mathrm{D}_{1}^{*+} \ell^{-} \bar{\nu}_{\ell}\right)$;

- $m_{D_{1}^{*}}$ : the mass of the $\mathrm{D}_{1}^{*}$;

- $\Gamma_{D_{1}^{*}}$ : the total width of the $\mathrm{D}_{1}^{*}$;

- $b_{D_{1}}=\mathrm{BR}\left(\overline{\mathrm{B}_{\mathrm{d}}^{0}} \rightarrow \mathrm{D}_{1}^{+} \ell^{-} \bar{\nu}_{\ell}\right)$;

- $b_{D_{2}^{*}}=\mathrm{BR}\left(\overline{\mathrm{B}_{\mathrm{d}}^{0}} \rightarrow \mathrm{D}_{2}^{*+} \ell^{-} \bar{\nu}_{\ell}\right)$

- $b_{N R}=\mathrm{BR}\left(\overline{\mathrm{B}_{\mathrm{d}}^{0}} \rightarrow \mathrm{D} \pi \ell^{-} \bar{\nu}_{\ell}\right)$ corresponding to a possible non-resonant contribution;

- $s_{N R}$ : the slope of an assumed mass distribution for the non-resonant component which is taken to be exponentially decreasing from threshold;

- $b_{\pi \pi}=\mathrm{BR}\left(\mathrm{D}^{* *} \rightarrow \mathrm{D} \pi \pi\right)$ which is assumed to be independent of any particular $\mathrm{D}^{* *}$ state.

Constraints from external measurements of the production rate and mass distribution for narrow states have been applied [33]:

- $b_{D_{1}}=(0.64 \pm 0.11) \%$;

- $b_{D_{2}^{*}}=(0.28 \pm 0.09) \%$.

Removing these two constraints from the fit and imposing the values for the mass and width of the broad $D_{1}^{*}$ state, we find $b_{D_{1}}=(0.33 \pm 0.17) \%$ and $b_{D_{2}^{*}}=(0.37 \pm 0.17) \%$, compatible with the values given above.

The following decay channels have been considered:

- $\mathrm{D}_{0}^{*} \rightarrow \mathrm{D} \pi$; 
- $\mathrm{D}_{1}^{*}$ and $\mathrm{D}_{1} \rightarrow \mathrm{D}^{*} \pi$ and $\mathrm{D} \rho$;

- the $\mathrm{D}_{2}^{*}$ can decay both into $\mathrm{D} \pi$ and $\mathrm{D}^{*} \pi$. The value $0.29 \pm 0.07$ [34] has been used for the decay probability into $\mathrm{D}^{*} \pi$ channels;

- the possible non-resonant component is expected to decay into $\mathrm{D} \pi$ only as, for the $\mathrm{D}^{*} \pi$ channel, there is no contribution from the $\mathrm{D}^{*}$ or $\mathrm{B}^{*}$ poles which are expected to play the main role in non-resonant production [24].

As this analysis is based on the reconstruction of charged particles, decay channels with neutrals appear at a lower mass than the genuine decaying $\mathrm{D}^{* *}$. Narrow states decaying into $\mathrm{D}^{*} \pi$, where the $\mathrm{D}^{*}$ decays into a neutral pion or photon and a reconstructed $\mathrm{D}$, still appear as peaks in the $\mathrm{D} \pi$ mass distribution, but slightly broader and displaced from their nominal values. The expected accuracy of the mass reconstruction for completely exclusive decays and the effects induced by missing neutrals have been studied using simulated events. For completely reconstructed $\mathrm{D}^{* *}$ decays, the experimental resolution on $\Delta_{m}$ is equal to $4 \mathrm{MeV} / c^{2}$. For decays with a $\mathrm{D}^{*}$ cascading into $\mathrm{D} \pi^{0}$ or $\mathrm{D} \gamma$, and in which the neutral particle is lost, an additional smearing of 4 or $15 \mathrm{MeV} / c^{2}$ is expected, respectively. For the $\mathrm{D}^{*+} \pi^{-}$exclusive decay channel, in which the $\mathrm{D}^{0}$, cascading from the $\mathrm{D}^{*+}$, decays into $\mathrm{K}^{-} \pi^{+}\left(\pi^{0}\right)$, the mass resolution is poorer and values of 50 and $70 \mathrm{MeV} / c^{2}$ have been obtained from the simulation for 94-95 and 92-93 data samples respectively. For $\mathrm{D}^{* *} \rightarrow \mathrm{D} \pi \pi$ decays, where only one pion is reconstructed, the $\mathrm{D} \pi$ mass distribution has been modelled using Gaussian distributions whose central value and standard deviation have been parametrized as a function of the central $\mathrm{D}^{* *}$ mass. Two parametrizations have been used for narrow and broad states, respectively. For broad states the variation of the standard deviation, as a function of the width of the $\mathrm{D}^{* *}$ state, has also been included. For narrow states with a $2.4 \mathrm{GeV} / c^{2}$ mass, the expected standard deviation of the $\mathrm{D} \pi$ mass distribution is $50 \mathrm{MeV} / c^{2}$ whereas it is $90 \mathrm{MeV} / c^{2}$ for a broad state having the same mass and a $200 \mathrm{MeV} / \mathrm{c}^{2}$ width.

Events have been distributed in two-dimensional histograms of the discriminant variable $R$ versus $\Delta_{m}$ with a $10 \mathrm{MeV} / c^{2}$ binning, to be sensitive to the presence of narrow components. For each channel $\left(\mathrm{D}^{0} \pi^{+}(2)^{7}, \mathrm{D}^{+} \pi^{-}(1)\right.$ and $\left.\mathrm{D}^{*+} \pi^{-}(3)\right)$ and each data set (2), corresponding to twelve histograms in total, the observed number of events in each bin has been compared with the expectations assuming a Poisson distribution with average corresponding to the sum of the expected number of signal and background events.

Two-dimensional distributions for the expected number of signal and background events have been constructed from the product of one-dimensional $\Delta_{m}$ and $R$ distributions.

For background events, the $\Delta_{m}$ distributions have been taken from simulation after verifying that their shape agreed with those measured with real data events selected in the sidebands (see Figure 5). A fit of these distributions, using the parametrization given by the following expression:

$$
\operatorname{Bckg}\left(\Delta_{m}\right)=\left(\Delta_{m}-m_{\pi}\right)^{\alpha} \exp \left(p_{0}+p_{1} \Delta_{m}+p_{2} \Delta_{m}^{2}+p_{3} \Delta_{m}^{3}+p_{4} \Delta_{m}^{4}\right) ; \alpha=0.5,
$$

has been performed and the fitted distribution has been used to determine the expected average background in each bin. The $R$ distribution corresponds to the one resulting from the fit of the different background components done in Section 3.3. The normalisation of background events has also been obtained from these fits.

The $\Delta_{m}$ distributions for signal events have been obtained by summing all components whose contribution is expected in the various channels. Breit-Wigner distributions have been used for each resonant state $(i)$ :

${ }^{7}$ The numbers in parentheses indicate the number of analysed samples corresponding to different $\mathrm{D}^{(0,+)}$ decay channels. 


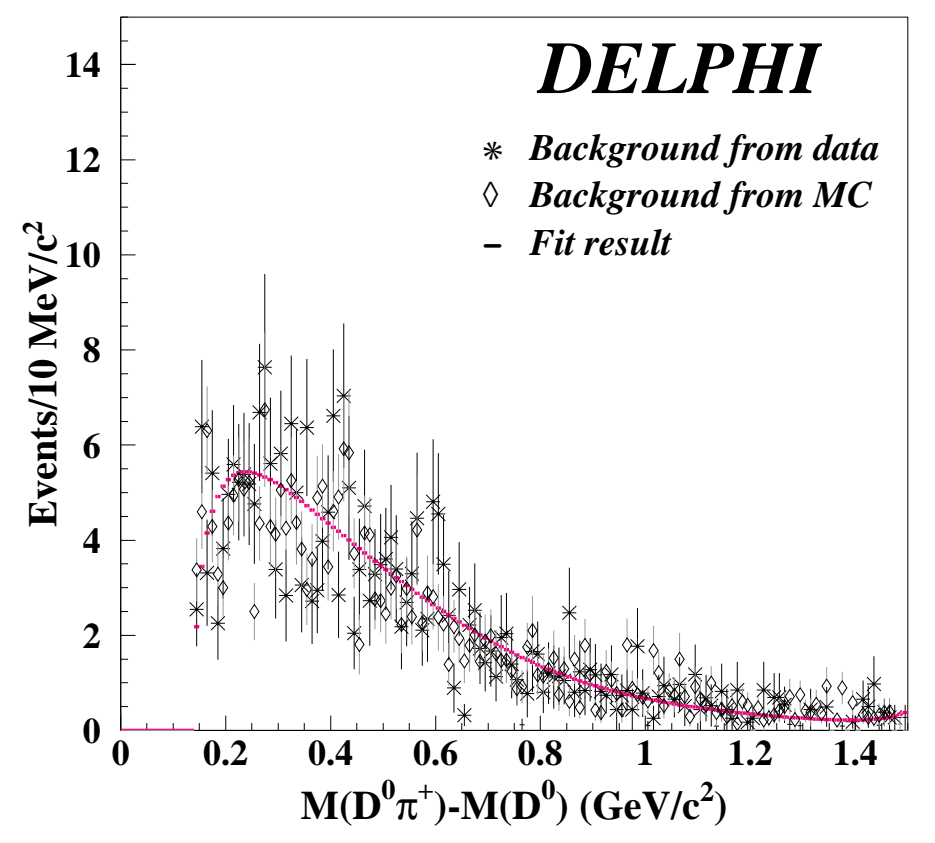

Figure 5: Comparison between $\Delta_{m}$ distributions obtained for combinatorial background events selected in the sidebands for real data (stars) and simulation (diamonds) corresponding to the channel $\mathrm{D}^{0} \pi^{+}$in the 1994-1995 data sample. The two distributions have been normalized to the same number of entries. The curve fitted to simulated events has been superimposed. 


$$
B W\left(\Delta_{m}\right)=N_{i} \frac{\frac{\Gamma_{i j}}{2}}{\left(m-m_{i}\right)^{2}+\left(\frac{\Gamma_{i j}}{2}\right)^{2}} .
$$

In this expression, $m$ is equal to $m\left(\mathrm{D}^{(*)}\right)+\Delta_{m}+$ shift $_{j}$ with shift $_{j}$ parametrizing the possible displacement due to loss of neutrals in the $\mathrm{D}^{*}$ decay, depending on the channel $j$ of interest. The width, $\Gamma_{i j}$, receives contributions from both the natural width of the physical state $\Gamma_{i}$ and from the experimental resolution. Expected variations of $\Gamma_{i}$, as a function of the mass $(m)$ and of the angular momentum in the decay have been taken into account. Finally, $N_{i}$ is the normalisation factor for the integral of $B W\left(\Delta_{m}\right)$ over the accessible mass range to be unity.

For the non-resonant $\mathrm{D} \pi$ component, a normalized exponential distribution has been used:

$$
N R\left(\Delta_{m}\right)=\frac{s_{N R} \exp \left(-s_{N R}\left(\Delta_{m}-m_{\pi}\right)\right)}{1-\exp \left(-s_{N R}\left(\Delta_{m}^{\max }-m_{\pi}\right)\right)}
$$

This distribution is maximum for low values of $\Delta_{m}$. Such a behaviour is expected when considering that the non-resonant $\mathrm{D} \pi$ component is induced by the $\mathrm{D}^{*}$ and $\mathrm{B}^{*}$ poles, using chiral dynamics [24]. Using the same model, in the $\mathrm{D}^{*} \pi$ decay channel, the nonresonant contribution is expected to be small, as the $\mathrm{D}^{*}$ and $\mathrm{B}^{*}$ poles are not effective in this channel, and it has been neglected.

The $R$ distribution for signal events is taken from the simulation.

\subsection{Results on production characteristics of $\mathrm{D}^{* *}$ states}

Results of the fit are given in Table 10. The corresponding $\Delta_{m}$ mass distributions are shown in Figure 6 and the fitted $D^{* *}$ mass distribution, comprising all components, is given in Figure 7.

Among the fitted parameters, three sets can be considered:

- quantities corresponding to significant measurements in the present analysis: the production rate, mass and width of the broad $\mathrm{D}_{1}^{*}$ state;

- quantities introduced to parametrize components which are possibly contributing in the hadronic final state, but for which the present statistics do not allow a significant measurement. These components have been introduced as they can correspond to different measured $\mathrm{D}^{(*)} \pi$ mass distributions. For instance it is not equivalent to consider a measured $\mathrm{D} \pi$ mass as originating from the non-resonant component or from a $\mathrm{D} \pi \pi$ decay. These parameters are the $\mathrm{D}_{0}^{*}$ production rate and width and the two quantities describing the possible non-resonant component;

- quantities constrained by external measurements, in the fit, such as the production fractions of narrow $\mathrm{D}_{1}$ and $\mathrm{D}_{2}^{*}$ states.

In addition, two parameters $\left(m_{D_{0}^{*}}\right.$ and $\left.b_{\pi \pi}\right)$ have been kept fixed in the fit and then varied within a specified range to evaluate their contribution to systematic uncertainties. In the following some remarks on these results are made.

\subsubsection{Broad states $D_{0}^{*}$ and $D_{1}^{*}$}

Most of the $\mathrm{D}^{*} \pi$ component originates from the broad $\mathrm{D}_{1}^{*}$ state whose mass and width have been measured. These two last values can be compared with results obtained for this particle by the CLEO [35] and BELLE [36] collaborations, using $\mathrm{B} \rightarrow \mathrm{D}^{(*)} \pi \pi$ decays: 

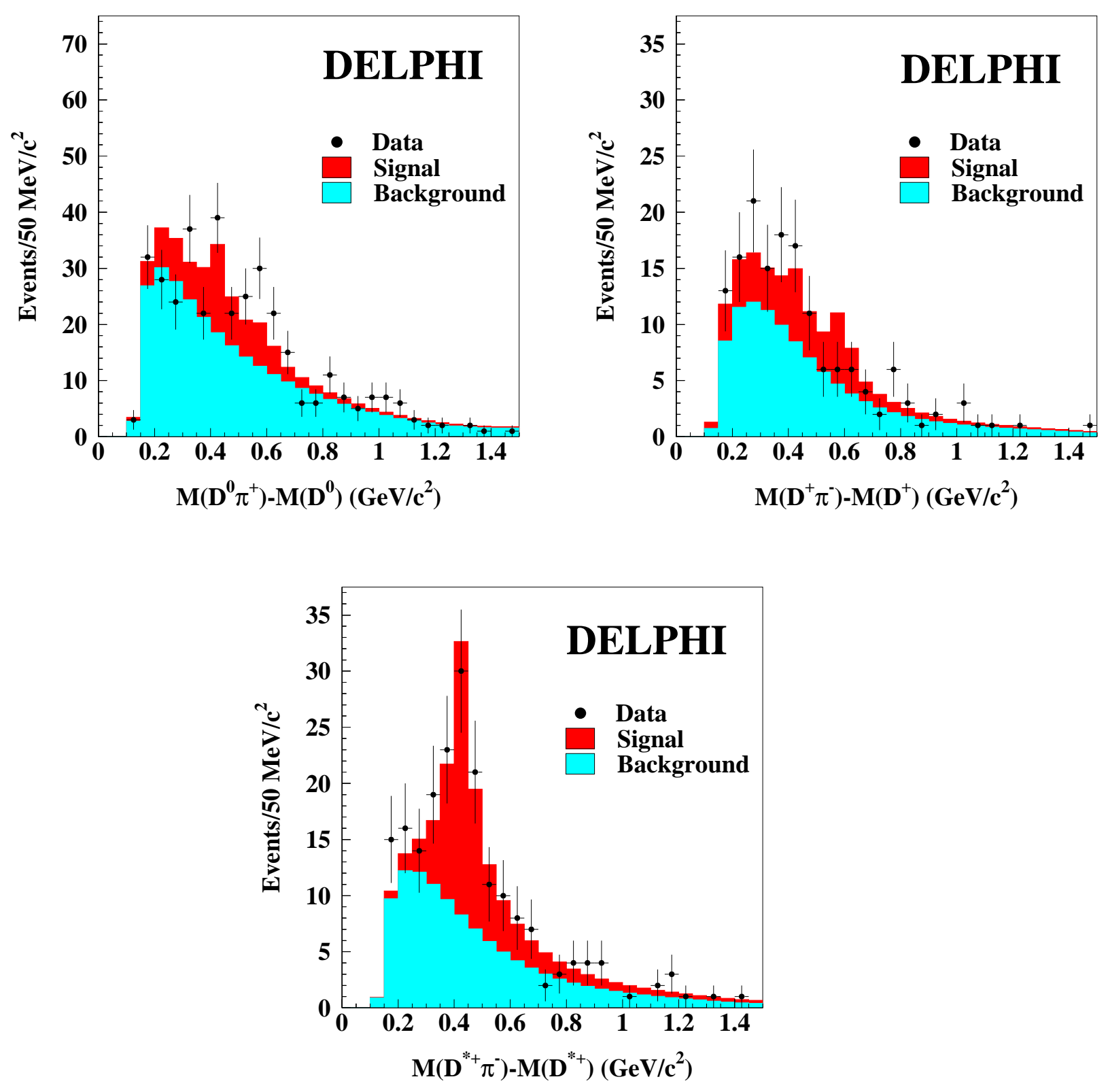

Figure 6: $\Delta_{m}$ distributions for right-sign events corresponding to the three $\mathrm{D}^{(*)} \pi$ combinations. The fitted signal contributions are superimposed. 




Figure 7: $\mathrm{D}^{* *}$ fitted mass distribution. The different components are shown within bands corresponding to the uncertainties of the fitted parameters.

$$
\begin{array}{cc}
m_{D_{1}^{*}}=2461_{-34}^{+41} \pm 10 \pm 32 \mathrm{MeV} / c^{2} ; & \Gamma_{D_{1}^{*}}=290_{-79}^{+101} \pm 26 \pm 36 \mathrm{MeV} / c^{2}[35] \\
m_{D_{1}^{*}}=2427 \pm 26 \pm 20 \pm 15 \mathrm{MeV} / c^{2} ; & \Gamma_{D_{1}^{*}}=384_{-75}^{+107} \pm 24 \pm 70 \mathrm{MeV} / c^{2}[36]
\end{array}
$$

This is the first time that the parameters of this resonance have been measured in semileptonic B hadron decays.

The present statistics is not sufficient to evaluate separately the contributions from the $\mathrm{D}_{0}^{*}$ (or other broad resonances), from the non-resonant component and from a possible $\mathrm{D} \pi \pi$ decay mode of $\mathrm{D}^{* *}$ states.

\subsubsection{The non-resonant component}

The fitted rate of the non-resonant component is compatible with zero and this is in agreement with expectations. The contribution from the $\mathrm{D}^{*}$ pole to the non-resonant $\mathrm{D} \pi$ component can be evaluated knowing the total branching fraction for the decay $\overline{\mathrm{B}_{\mathrm{d}}^{0}} \rightarrow \mathrm{D}^{*+} \ell^{-} \bar{\nu}_{\ell}$, the minimal value used for $\Delta_{m}$ to select the events and the value of the $\mathrm{D}^{*}$ width in the hypothesis that the mass is distributed according to a Breit-Wigner:

$$
\begin{aligned}
\mathrm{BR}\left(\overline{\mathrm{B}_{\mathrm{d}}^{0}}\right. & \left.\rightarrow \mathrm{D}^{0} \pi^{+} \ell^{-} \bar{\nu}_{\ell}\right)\left.\right|_{N R} ^{\mathrm{D}^{*} \text { pole }} \\
& =\frac{2}{3}\left(1-\frac{2}{\pi} \arctan 2 \frac{\left.m\left(\mathrm{D}^{0} \pi^{+}\right)\right|_{m i n .}-m\left(\mathrm{D}^{*+}\right)}{\Gamma_{\mathrm{D}^{*+}}}\right) \times \mathrm{BR}\left(\overline{\mathrm{B}_{\mathrm{d}}^{0}} \rightarrow \mathrm{D}^{*+} \ell^{-} \bar{\nu}_{\ell}\right) \simeq 0.12 \%
\end{aligned}
$$

corresponding to a minimum $\mathrm{D}^{0} \pi^{+}$mass situated at 10 widths from the pole. For $\left.\mathrm{BR}\left(\mathrm{B}^{-} \rightarrow \mathrm{D}^{+} \pi^{-} \ell^{-} \bar{\nu}_{\ell}\right)\right|_{N R} ^{\mathrm{D}^{*} \text { pole }}$, there is a natural cutoff corresponding to the sum of the $\mathrm{D}^{+}$and $\pi^{-}$masses and the value becomes $0.05 \%$. 


\begin{tabular}{|c|c|}
\hline parameter & $\begin{array}{c}\text { Fitted value } \\
\text { stat. err. } \pm \text { syst. }\end{array}$ \\
\hline$b_{D_{1}^{*}}$ & $(1.24 \pm 0.25 \pm 0.27) \%$ \\
$m_{D_{1}^{*}}$ & $2445 \pm 34 \pm 10 \mathrm{MeV} / c^{2}$ \\
$\Gamma_{D_{1}^{*}}$ & $234 \pm 74 \pm 25 \mathrm{MeV} / c^{2}$ \\
\hline$b_{D_{0}^{*}}$ & $(0.42 \pm 0.33 \pm 0.22) \%$ \\
$\Gamma_{D_{0}^{*}}$ & $260 \pm 130 \pm 130 \mathrm{MeV} / c^{2}$ \\
$b_{N R}$ & $(0.23 \pm 0.35 \pm 0.44) \%$ \\
$s_{N R}$ & $5 \pm 7\left(\mathrm{GeV} / c^{2}\right)^{-1}$ \\
\hline$b_{D_{1}}$ & $(0.56 \pm 0.10) \%($ constrained) \\
$b_{D_{2}^{*}}$ & $(0.30 \pm 0.08) \%$ (constrained) \\
\hline
\end{tabular}

Table 10: Fitted values for parameters of the $\mathrm{D}^{* *}$ production characteristics.

The contribution from the $\mathrm{B}^{*}$ pole can be also included in a controlled way by considering that the slow pion emission, by mesons containing a heavy quark, is governed by a universal parameter $\hat{g}$ whose value is fixed by the measured $\mathrm{D}^{*}$ width [37]:

$$
\Gamma\left(\mathrm{D}^{*} \rightarrow \mathrm{D} \pi\right)=\frac{\hat{g}^{2}}{4 \pi f_{\pi}^{2}} p_{\pi}^{3}
$$

where $f_{\pi}$ is the pion decay constant and $p_{\pi}$ is the momentum in the $\mathrm{D}^{*}$ rest frame. The interference term between the $\mathrm{D}^{*}$ and $\mathrm{B}^{*}$ pole contributions is also fixed and, using the values given above, this gives:

$$
\left.\mathrm{BR}\left(\overline{\mathrm{B}_{\mathrm{d}}^{0}} \rightarrow \mathrm{D}^{0} \pi^{+} \ell^{-} \bar{\nu}_{\ell}\right)\right|_{N R} ^{\mathrm{D}^{*}+\mathrm{B}^{*} \text { poles }} \simeq 0.07 \%
$$

The main uncertainty, in the non-resonant contribution, comes from the interference with the $\mathrm{D}_{0}^{*}$. Varying the branching fraction, $\mathrm{BR}\left(\overline{\mathrm{B}_{\mathrm{d}}^{0}} \rightarrow \mathrm{D}_{0}^{*} \ell^{-} \bar{\nu}_{\ell}\right)$ between 0.1 and $0.8 \%$, the non-resonant contribution changes between $+0.02 \%$ and $-0.09 \%$ assuming that the interference has a negative sign below the $\mathrm{D}_{0}^{*}$ pole. The corresponding variation becomes $+0.1 \%$ to $+0.2 \%$ for the other sign.

\subsubsection{Total $\mathrm{D}^{* *}$ production rate}

Summing all fitted components, the total rate for $\mathrm{D}^{* *}$ production amounts to:

$$
\mathrm{BR}\left(\overline{\mathrm{B}_{\mathrm{d}}^{0}} \rightarrow \mathrm{D}^{* *} \ell^{-} \bar{\nu}_{\ell}\right)=(2.7 \pm 0.7 \pm 0.2) \%
$$

\subsubsection{Systematic uncertainties}

In addition to those considered in Table 8, systematic uncertainties have been evaluated for the following effects:

- the $b_{\pi \pi}$ branching fraction has been varied within the range $(20 \pm 15) \%$. The central value corresponds to an estimate obtained by comparing the phase-space integrals for the $\mathrm{D}^{*} \pi$ and $\mathrm{D} \rho$ decay modes of a $\mathrm{D}^{* *}$ state. This variation is also in agreement with the value obtained in data for this parameter when it is left free to vary in the fit: $(19 \pm 13) \%$; 
- the central value of the $\mathrm{D}_{0}^{*}$ mass, $m_{D_{0}^{*}}$, which is kept fixed during the fit has been varied between 2.3 and $2.5 \mathrm{GeV} / c^{2}$;

- parameters used to define the shape of the combinatorial background distributions in $\Delta_{m}$ (see Equation (9)) have been varied. The value of $\alpha$ has been changed between 0.3 and 0.7 and the degree of the polynomial function was also taken as 3 or 5 ;

- the contribution from $\overline{\mathrm{B}_{\mathrm{s}}^{0}}$ semileptonic decays, which have been modelled similarly to the non-strange $b$-mesons. Properties of narrow $\mathrm{D}_{s}^{* *}$ states published in [21] have been used. For broad states it has been assumed that their masses were displaced, relative to the corresponding non-strange states by the same amount as for narrow states. $\Delta_{m}$ mass distributions for $\mathrm{D}^{(*)} \mathrm{K}^{+}$final states have also been evaluated by considering that the $\mathrm{K}^{+}$was reconstructed as a $\pi^{+}$. For excited charm states produced in semileptonic decays of the $\Lambda_{b}^{0}$, no simulation has been used, considering the lack of information on such decays. It has been assumed that the contribution from these final states was about $50 \%$ of the variation observed when $\overline{\mathrm{B}_{\mathrm{s}}^{0}}$ decays are introduced. An uncertainty corresponding to twice this variation has been used to account for $\overline{\mathrm{B}_{\mathrm{s}}^{0}}$ and $\Lambda_{b}^{0}$ contributions;

- considering the recent result from the BELLE collaboration which has measured a larger width for $\mathrm{D}_{2}^{*}$ mesons [36] than quoted in [21], the effect of changing this value from 20 to $40 \mathrm{MeV} / c^{2}$ has been evaluated.

Individual contributions from these sources of systematics have been listed when evaluating moments of the hadronic mass distribution. It has also been verified that possible additional systematic sources such as:

- the relative branching fraction of $\mathrm{D}_{2}^{*}$ mesons into $\mathrm{D}^{*} \pi$ which has been varied according to the expected value: $0.29 \pm 0.07$ [34];

- the expected mass reconstruction accuracy which has been varied by $30 \%$

have negligible effects on hadronic mass moments. Uncertainties related to the control of the shape of the discriminant variable distribution have a small effect (4\%) on the measured $\mathrm{D}^{* *}$ production rate and negligible contributions to hadronic mass moments.

\section{Moments of the hadronic mass distribution in $b$ - hadron semileptonic decays}

Moments of the $\mathrm{D}^{* *}$ mass distribution can be evaluated from the results of the fit discussed above. Results are given in Table 11. Statistical uncertainties have been obtained by propagating those on fitted parameters, using their full covariance matrix. Systematics are dominated by the uncertainty on the possible contribution from $\mathrm{D} \pi \pi$ decays and could be reduced in future when experimental results on this decay channel become available.

In determining the moments of the complete hadronic mass distribution in $b$-hadron semileptonic decays, $b \rightarrow \mathrm{D}$ and $\mathrm{D}^{*} \ell^{-} \bar{\nu}_{\ell}$ channels have been included.

For the first channel, values of branching fractions given in [21], have been used. As $\overline{\mathrm{B}_{\mathrm{d}}^{0}}$ and $\mathrm{B}^{-}$are expected to have the same partial decay width into the $\mathrm{D} \ell^{-} \bar{\nu}_{\ell}$ channel, we get:

$$
\mathrm{BR}\left(\overline{\mathrm{B}_{\mathrm{d}}^{0}} \rightarrow \mathrm{D}^{+} \ell^{-} \bar{\nu}_{\ell}\right)=(2.06 \pm 0.20) \% .
$$




\begin{tabular}{|c|c|c|c|c|c|c|}
\hline & $\begin{array}{c}<m_{D^{* *}}> \\
\left(\mathrm{GeV} / c^{2}\right)\end{array}$ & $\begin{array}{c}<m_{D^{* *}}^{2}> \\
\left(\mathrm{GeV} / c^{2}\right)^{2}\end{array}$ & $\begin{array}{c}<m_{D^{* *}}^{4}> \\
\left(\mathrm{GeV} / c^{2}\right)^{4}\end{array}$ & $\begin{array}{c}<m_{D^{* *}}^{6}> \\
\left(\mathrm{GeV} / c^{2}\right)^{6}\end{array}$ & $\begin{array}{c}<m_{D^{* *}}^{8}> \\
\left(\mathrm{GeV} / c^{2}\right)^{8}\end{array}$ & $\begin{array}{c}<m_{D^{* *}}^{10}> \\
\left(\mathrm{GeV} / c^{2}\right)^{10}\end{array}$ \\
\hline value & 2.483 & 6.22 & 40.1 & 270.6 & 1932 & 14732 \\
\hline stat. uncert. & \pm 0.033 & \pm 0.16 & \pm 2.0 & \pm 20.9 & \pm 206 & \pm 2039 \\
\hline$b_{\pi \pi}$ & \pm 0.030 & \pm 0.14 & \pm 1.6 & \pm 14.4 & \pm 122 & \pm 1032 \\
$m_{D_{0}^{*}}$ & \pm 0.008 & \pm 0.04 & \pm 0.3 & \pm 2.5 & \pm 18 & \pm 36 \\
backg. param & 0.003 & 0.02 & 0.2 & 2.6 & 28 & 291 \\
$\bar{B}_{\mathrm{s}}^{0}, \Lambda_{b}^{0}$ & \pm 0.010 & \pm 0.04 & \pm 0.5 & \pm 4.3 & \pm 38 & \pm 334 \\
casc. rate & 0.001 & 0.01 & 0.1 & 0.4 & 4 & 25 \\
$d_{ \pm}$dist. & 0.002 & 0.01 & 0.2 & 2.0 & 22 & 244 \\
$\Gamma\left(D_{2}^{*}\right)=40 \mathrm{MeV} / c^{2}$ & -0.002 & -0.01 & -0.2 & -2.0 & -19 & -200 \\
\hline Tot. syst. & 0.033 & 0.15 & 1.7 & 15.7 & 135 & 1167 \\
\hline
\end{tabular}

Table 11: Measured moments of the $\mathrm{D}^{* *}$ mass distribution. When the sign of the variation is not given this is because the corresponding quoted systematic error originates from several sources corresponding to different signs which are given in the text.

For the second channel, the value given in [38] at the time of the Winter 2003 conferences has been used:

$$
\mathrm{BR}\left(\overline{\mathrm{B}_{\mathrm{d}}^{0}} \rightarrow \mathrm{D}^{*+} \ell^{-} \bar{\nu}_{\ell}\right)=(5.27 \pm 0.19) \%
$$

The inclusive semileptonic branching fraction $\left(\mathrm{BR}\left(\overline{\mathrm{B}_{\mathrm{d}}^{0}} \rightarrow c \ell^{-} \bar{\nu}_{\ell}\right)\right)$ has also been included as a constraint:

$$
\mathrm{BR}\left(\overline{\mathrm{B}_{\mathrm{d}}^{0}} \rightarrow c \ell^{-} \bar{\nu}_{\ell}\right)=\frac{\tau\left(\overline{\mathrm{B}_{\mathrm{d}}^{0}}\right)}{\tau(b)} \operatorname{BR}\left(b \rightarrow c \ell^{-} \bar{\nu}_{\ell}\right)=(10.25 \pm 0.30) \%
$$

This last value has been obtained using the average values determined at LEP for the quantities entering into Equation (16), which are given in Tables 7 and 18. As the inclusive semileptonic branching fraction is the sum of the $\mathrm{D}, \mathrm{D}^{*}$ and $\mathrm{D}^{* *}$ contributions, the expected rate for $\mathrm{D}^{* *}$ production can be derived from these values:

$$
\mathrm{BR}\left(\overline{\mathrm{B}_{\mathrm{d}}^{0}} \rightarrow \mathrm{D}^{* *} \ell^{-} \bar{\nu}_{\ell}\right)=(2.9 \pm 0.4) \%
$$

This is in agreement with the rate measured directly as given in Equation (13).

Moments of the hadronic mass distribution have then been derived as:

$$
<m_{H}^{n}>=p_{D} m_{D}^{n}+p_{D^{*}} m_{D^{*}}^{n}+p_{D^{* *}}<m_{D^{* *}}^{n}>
$$

where $p_{D_{i}}=\frac{\mathrm{BR}\left(\overline{\mathrm{B}_{\mathrm{d}}^{0}} \rightarrow \mathrm{D}_{i} \ell^{-} \bar{\nu}_{\ell}\right)}{\mathrm{BR}\left(\overline{\mathrm{B}_{\mathrm{d}}^{0}} \rightarrow c \ell^{-} \bar{\nu}_{\ell}\right)}$. The value of $p_{D^{* *}}$ has been obtained by imposing the constraint $1=p_{D}+p_{D^{*}}+p_{D^{* *}}$ and including the measurement of the $\mathrm{D}^{* *}$ production rate as given in Equation (13).

Results, following the notations in Equation (3), are given in Tables 12 and 13. Systematic uncertainties related to measurements of the branching fractions in Equations (14, 16) are also given in Tables 12 and 13.

Effects induced by the variation of the analysis efficiency versus the mass of $\mathrm{D}^{* *}$ states have been evaluated to correspond to an increase of $0.7,1.5$ and $2.5 \%$ for $M_{1}^{H}, M_{2}^{\prime H}$ and $M_{3}^{\prime H}$ respectively. Considering the present level of statistical and systematic uncertainty of actual measurements, these corrections have not been included in the quoted central values for hadronic moments.

Error correlation matrices are given in Appendix A. 


\begin{tabular}{|c|c|c|c|c|c|}
\hline & $\begin{array}{c}M_{1}^{H} \\
\left(\mathrm{GeV} / c^{2}\right)^{2}\end{array}$ & $\begin{array}{c}M_{2}^{H} \\
\left(\mathrm{GeV} / c^{2}\right)^{4}\end{array}$ & $\begin{array}{c}M_{3}^{H} \\
\left(\mathrm{GeV} / c^{2}\right)^{6}\end{array}$ & $\begin{array}{c}M_{4}^{H} \\
\left(\mathrm{GeV} / c^{2}\right)^{8}\end{array}$ & $\begin{array}{c}M_{5}^{H} \\
\left(\mathrm{GeV} / c^{2}\right)^{10}\end{array}$ \\
\hline value & 0.647 & 1.98 & 7.4 & 35.7 & 205 \\
\hline stat. uncert. & \pm 0.046 & \pm 0.23 & \pm 1.3 & \pm 7.9 & \pm 1080 \\
\hline Ext. BR & 0.079 & 0.22 & 0.8 & 4.1 & 23.4 \\
$b_{\pi \pi}$ & \pm 0.039 & \pm 0.15 & \pm 0.6 & \pm 2.8 & \pm 16.4 \\
$m_{D_{0}^{*}}$ & \pm 0.015 & \pm 0.04 & $\mp 0.0$ & $\mp 0.7$ & $\mp 7.0$ \\
backg. param & 0.007 & 0.04 & 0.2 & 1.2 & 8.0 \\
$\overline{\mathrm{B}}_{\mathrm{s}}^{0}, \Lambda_{b}^{0}$ & \pm 0.007 & \pm 0.03 & \pm 0.2 & \pm 0.9 & \pm 5.2 \\
$d_{ \pm}$dist. & 0.005 & 0.03 & 0.2 & 1.1 & 7.4 \\
$\Gamma\left(D_{2}^{*}\right)=40 \mathrm{MeV} / c^{2}$ & -0.004 & -0.02 & -0.1 & -0.9 & -5.8 \\
\hline Tot. syst. & 0.090 & 0.27 & 1.1 & 5.4 & 32.3 \\
\hline
\end{tabular}

Table 12: Measured moments of the hadronic mass distribution, by reference to the spin averaged D-hadron mass, in b-hadron semileptonic decays.

\begin{tabular}{|c|c|c|c|c|}
\hline & $\begin{array}{c}M_{2}^{\prime H} \\
\left(\mathrm{GeV} / c^{2}\right)^{4}\end{array}$ & $\begin{array}{c}M_{3}^{\prime H} \\
\left(\mathrm{GeV} / c^{2}\right)^{6}\end{array}$ & $\begin{array}{c}M_{4}^{\prime H} \\
\left(\mathrm{GeV} / c^{2}\right)^{8}\end{array}$ & $\begin{array}{c}M_{5}^{\prime H} \\
\left(\mathrm{GeV} / c^{2}\right)^{10}\end{array}$ \\
\hline value & 1.56 & 4.05 & 21.1 & 116.0 \\
\hline stat. uncert. & \pm 0.18 & \pm 0.74 & \pm 4.5 & \pm 27.0 \\
\hline Ext. BR & 0.12 & 0.15 & 1.1 & 5.0 \\
$b_{\pi \pi}$ & \pm 0.10 & \pm 0.17 & \pm 1.0 & \pm 5.2 \\
$m_{D_{0}^{*}}$ & \pm 0.02 & $\mp 0.10$ & $\mp 0.8$ & $\mp 6.0$ \\
backg. param & 0.03 & 0.12 & 0.7 & 4.2 \\
$\bar{B}_{\mathrm{s}}^{0}, \Lambda_{b}^{0}$ & \pm 0.02 & \pm 0.06 & \pm 0.4 & \pm 2.3 \\
$d_{ \pm}$dist. & 0.02 & 0.11 & 0.7 & 4.0 \\
$\Gamma\left(D_{2}^{*}\right)=40 \mathrm{MeV} / c^{2}$ & -0.02 & -0.10 & -0.5 & -3.1 \\
\hline Tot. syst. & 0.16 & 0.32 & 2.1 & 11.7 \\
\hline
\end{tabular}

Table 13: Measured moments of the hadronic mass distribution, by reference to the average mass squared, in b-hadron semileptonic decays.

\section{Moments of the lepton energy distribution in $b$ - hadron semileptonic decays}

\subsection{Inclusive reconstruction of $b$-hadron semileptonic decays}

Selected events have been divided into two hemispheres using the thrust axis. The secondary hadronic system accompanying the lepton in the semileptonic decay has been reconstructed using an iterative procedure applied to the particles belonging to the same hemisphere as the tagged lepton.

Charged particles, belonging to the hemisphere of the candidate lepton, with $p>0.7$ $\mathrm{GeV} / c$, and with at least one associated hit in the Vertex Detector and positive impact parameter with respect to the primary vertex have been considered. These have been sorted in decreasing order of their probability of being B decay products based on their impact parameter significance and considering the particle that crosses the jet direction the furthest away from the primary vertex, or, when the crossing is not well-defined, the most energetic particle, to be the most likely B decay product.

The charged particles have been iteratively tested for forming a secondary vertex. The procedure has been iterated while the following conditions have been fulfilled: invariant mass below $2.9 \mathrm{GeV} / c^{2}$, distance from the primary vertex less than $3 \mathrm{~cm}$ but at least 
2.8 times the uncertainty and on the positive side, and $\Delta \chi^{2}<3$ after inclusion of each particle in the seed vertex fit.

In those cases where no secondary vertex was found, single particles have been accepted when fulfilling one of the following criteria, in decreasing order of quality: a charged particle with $p>3 \mathrm{GeV} / c$ having a crossing point with the jet axis at least $1 \sigma$ downstream from the primary vertex and also downstream from the lepton candidate crossing point, but less than $15 \mathrm{~cm}$ from the primary vertex; a charged particle with $p>3 \mathrm{GeV} / c$ with the largest impact parameter significance and positive impact parameter sign; the most energetic charged particle within $0.6 \mathrm{rad}$ of the jet axis.

The remaining charged particles in the hemisphere of the lepton with $p>0.5 \mathrm{GeV} / c$ and at least one vertex detector hit not yet associated with the secondary vertex are then considered. Each of them is tested to belong to the vertex, and the one with the smallest $\chi^{2}$ contribution and giving a vertex mass closest to the $\mathrm{D}$ mass is included in the vertex, and all the remaining particles are then tested against this new vertex. This process is continued until the remaining particles have large contributions to the vertex $\chi^{2}$ and increase the mass difference between the vertex and the $\mathrm{D}$ mass.

To improve the purity of the vertex, each of the particles associated with it is reconsidered, if there are more than two particles in the vertex, the $\chi^{2} /$ n.d.f of the vertex exceeds 2 and the mass of the vertex is more than the D mass. If removing a particle from the vertex improves the $\chi^{2} /$ n.d.f and the mass after the removal is closer to the $\mathrm{D}$ mass, the particle is permanently removed from the vertex. The average charged multiplicity of the vertices is 2.9. As the last stage, identified $K_{s}^{0} \mathrm{~s}$ within $0.8 \mathrm{rad}$ and $\pi^{0} \mathrm{~s}$ within $1 \mathrm{rad}$ of the lepton direction have been tested for association based on their energy, rapidity and contribution to the vertex mass.

On average, $78 \%$ of the particles associated with the vertex were true $\mathrm{D}$ decay products, and $74 \%$ of the decay products were correctly associated with the vertex. The mass distribution of the reconstructed vertex is shown in Figure 8.

The hadronic energy is obtained from the energy of the reconstructed secondary vertex, corrected with a linear function of the reconstructed vertex mass, when the reconstructed mass is below the $\mathrm{D}^{0}$ meson mass. The direction of the hadronic system is taken from the momentum sum of the particles included in the vertex.

For each decay, the energy of the B hadron has been estimated as the energy sum of the secondary hadronic system, the identified lepton and the neutrino energy.

The energy obtained in this way was corrected by a function of the reconstructed hadronic energy, determined from simulation, with the maximum correction being $\pm 6 \mathrm{GeV}$. The neutrino energy was computed from the missing energy and momentum in both hemispheres corrected by a function of the missing mass in the event, determined from simulation. The resolution of the neutrino energy in $\mathrm{B} \rightarrow \mathrm{X} \ell \bar{\nu}_{\ell}$ decays was estimated to be $\pm 2.9 \mathrm{GeV}$. Neutrino energies larger than $1 \mathrm{GeV}$ were required.

The resulting resolution of the B energy was found to be $12 \%$ for $80 \%$ of all inclusive semileptonic B decays and $24 \%$ for the remaining decays. The resolution on the missing energy and on the reconstructed $\mathrm{B}$ hadron energy estimated with simulation for signal $\mathrm{B} \rightarrow \mathrm{X}_{c} \ell \bar{\nu}_{\ell}$ events are shown in Figure 9 .

The direction of the momentum vector of the reconstructed $\mathrm{X} \ell \bar{\nu}_{\ell}$ system was adjusted by up to $\pm 30 \mathrm{mrad}$ with respect to the lepton direction using a function of the reconstructed B mass. Another estimate for the B hadron direction was obtained from the $\mathrm{B}$ line of flight reconstructed from the position of the vertex formed by the lepton with the identified charm charged decay products. An estimator, which combines these two independent measurements according to their expected resolutions as a function of the 


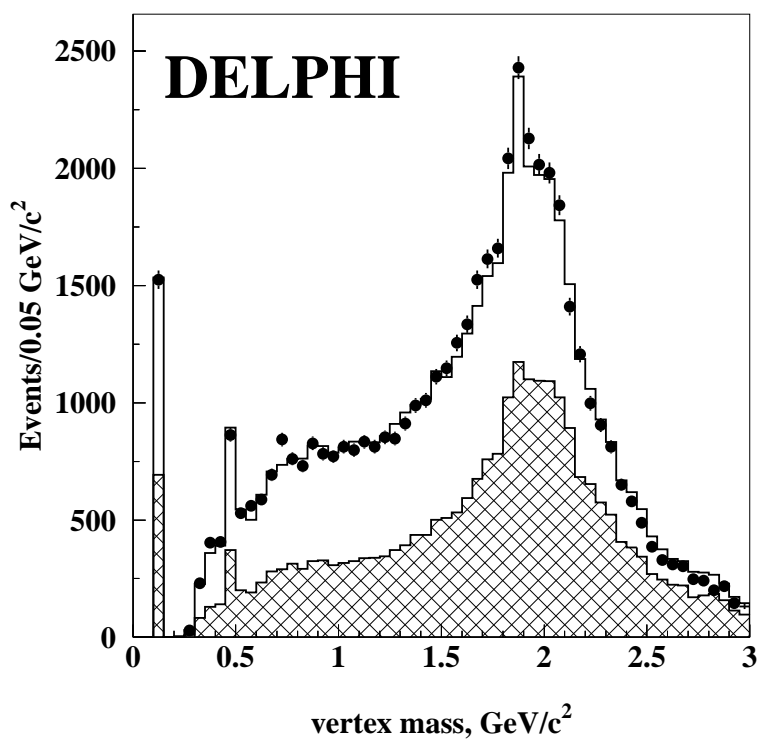

Figure 8: Mass distribution of the reconstructed charm vertex. The peaks at 0.14 and $0.5 \mathrm{GeV} / \mathrm{c}^{2}$ correspond to vertices consisting of a single $\pi$ or $K$, respectively. Points are the data, while the histogram is the simulation with the signal clear and the background hatched.

reconstructed energies and the decay distance respectively, was defined. Resolutions of $14(15) \mathrm{mrad}$ have been achieved in $\phi(\theta)$ for $60 \%$ of all inclusive semileptonic B decays and $40 \mathrm{mrad}$ for the remaining decays.

The identified lepton was then boosted back to the reconstructed B rest frame and its energy $E_{\ell}^{*}$ computed in this frame. This resulted in an average resolution of $170 \mathrm{MeV}$ on $E_{\ell}^{*}$ for $82 \%$ of all inclusive semileptonic B decays and $510 \mathrm{MeV}$ for the remaining decays.

\subsection{Signal separation from background sources}

In the reduction of the $b \rightarrow c \rightarrow \ell$ and other backgrounds it is essential to avoid biases of the lepton energy spectrum. The separation was therefore performed using two discriminating variables, one based on the topology of the event and the other on charge correlations between the lepton and the other particles in the event, which are not sensitive to the lepton energy.

The topological variable uses information on the lepton impact parameter with respect to the reconstructed secondary vertex, the topology of the tracks other than the lepton in the hemisphere, the number of particles not associated with the vertex in the hemisphere, the number of particles in the vertex and the $\chi^{2}$ of the vertex.

The charge variable consists of a probability built from the correlation of the charge of the lepton and those of the reconstructed secondary vertex, of other vertices in the same and opposite hemispheres, of the jet charge of the opposite hemisphere and of the leading kaon candidate. The kaon candidate was identified based on kaon neural network output from the MACRIB package [39]. Two-dimensional distributions are shown in 

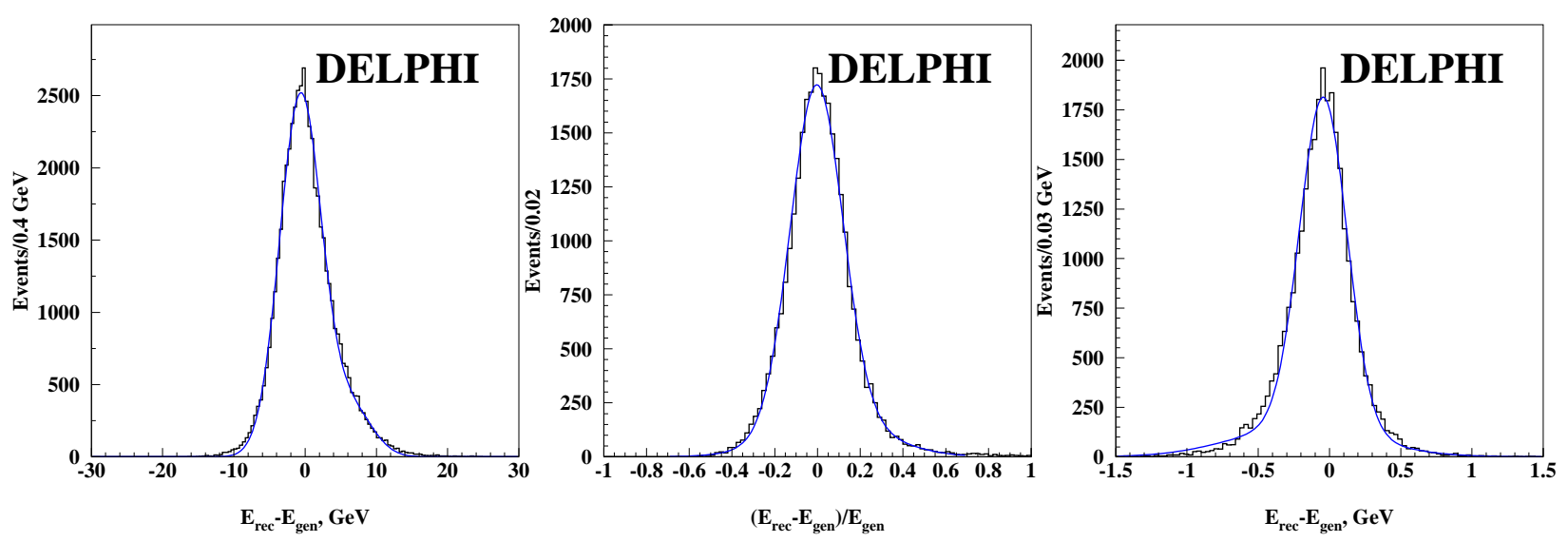

Figure 9: The resolution on the missing energy (left), the fractional resolution on the reconstructed $B$ hadron energy (centre) and resolution on the $E_{\ell}^{*}$ energy (right) estimated with simulation for signal $\mathrm{B} \rightarrow \mathrm{X}_{c} \ell \bar{\nu}_{\ell}$ events.

Figure 10 for signal and background, respectively. The final separation variable (VSEP) corresponds to the likelihood of an event to be a signal event, based on the location in the two-dimensional distribution of the topological and charge correlation variables.

A further rejection of background is obtained by requiring a minimum value for the reconstructed $\mathrm{B}$ mass. In Figure 11 the distribution of the $\mathrm{B}$ mass is shown in data and simulation, for signal and background events. A B mass larger than $3.9 \mathrm{GeV} / c^{2}$ was required. A final sample of 14364 leptons was selected, with a purity in $\mathrm{B} \rightarrow \mathrm{X}_{c} \ell \bar{\nu}_{\ell}$ decays of $81 \%$.

Figure 12 shows the lepton spectrum, after VSEP and B mass cuts, and the corresponding efficiencies as a function of $E_{\ell}^{*}$. A lepton sample, depleted in signal by using an anti-cut on VSEP is also shown, as a check of the shape of the simulated backgrounds with the data.

\subsection{Study of the lepton energy distribution}

The original lepton spectrum has been extracted from the reconstructed distribution by a spectrum re-weighting technique. This consisted of determining the resolution matrix relating the generated to the reconstructed spectrum for simulated signal events. Using this matrix, the coefficients of a re-weighting function for the generated spectrum have been fitted to minimize the $\chi^{2}$ between the resulting spectrum and that observed in the data. The efficiency correction has been taken into account at this stage. The procedure has been carefully tested on lepton spectra generated for different values of the $m_{b}$ and $\mu_{\pi}^{2}$ parameters and smeared according to the resolution matrix.

In order to increase the statistics in the signal description, the sample of simulated $b \bar{b}$ events (see Table 1) has also been used in the construction of the resolution matrix.

A regularized unfolding method [40] has also been applied as a cross-check, but the re-weighting method has been preferred for its simplicity.

\subsection{Results on lepton spectra}

The resulting lepton spectrum is shown in Figure 13. 


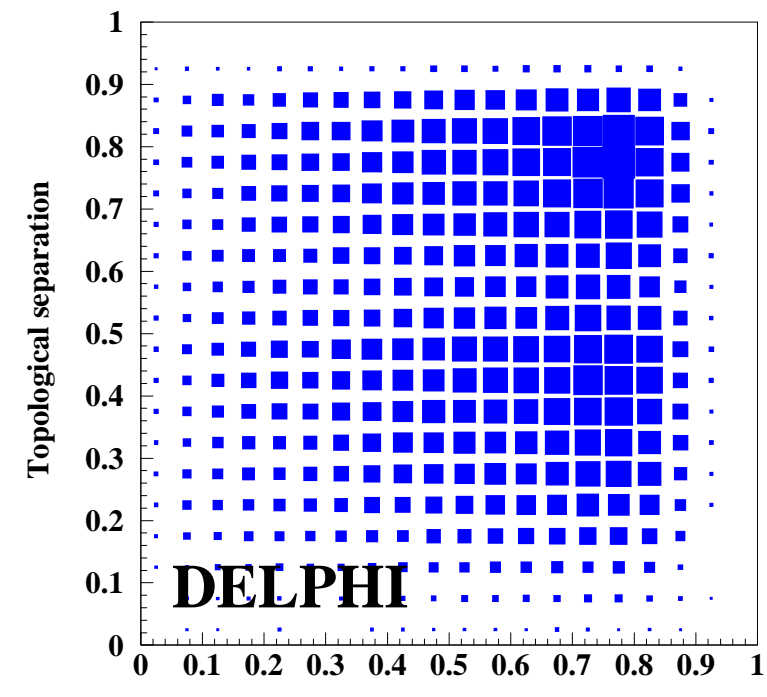

Charge separation

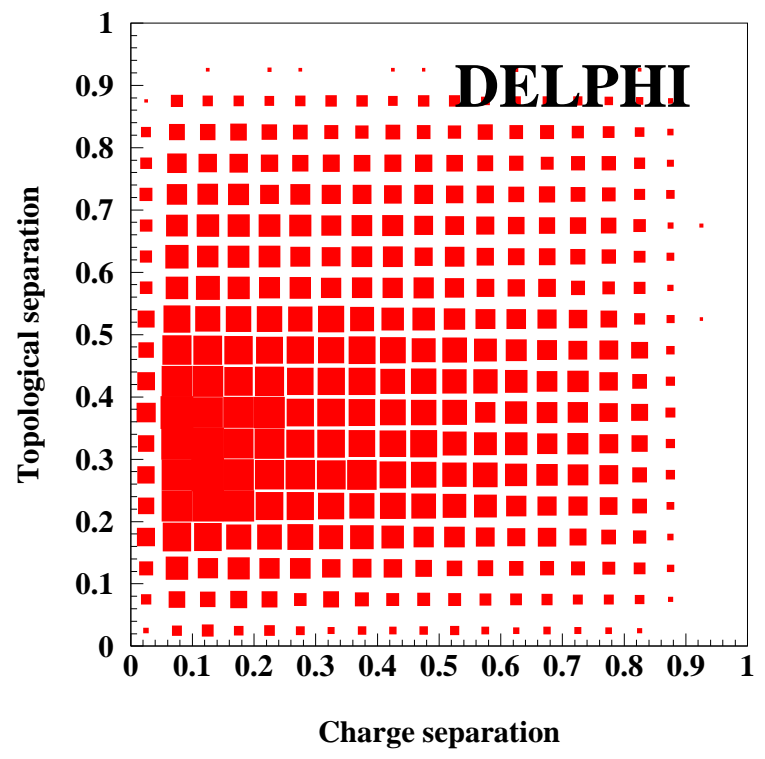

Charge separation

Figure 10: Two-dimensional distribution of the topological and charge correlation variables for the signal $\mathrm{B} \rightarrow \mathrm{X}_{c} \ell \bar{\nu}_{\ell}$ (left) and the main background $\mathrm{B} \rightarrow \mathrm{X}_{c} \rightarrow X \ell \nu$ (right).



Figure 11: Reconstructed B mass. Points are the data, histogram is the simulation with the signal clear and the background hatched. 



Figure 12: The resulting $E_{\ell}^{*}$ spectrum for samples enriched (left) and depleted (right) in $\mathrm{B} \rightarrow \mathrm{X}_{c} \ell \bar{\nu}_{\ell}$ decays using the separating variable. The data are shown as crosses while the simulation is shown as histograms. The different sources of background, shown shaded, are from bottom to top: $b \rightarrow \bar{c} \rightarrow \ell, b \rightarrow c \rightarrow \ell$, other lepton sources including decays in flight and converted photons, $c \rightarrow \ell$, misidentified hadrons. In the lower plots the efficiencies for the selection are shown, as a function of $E_{\ell}^{*}$.
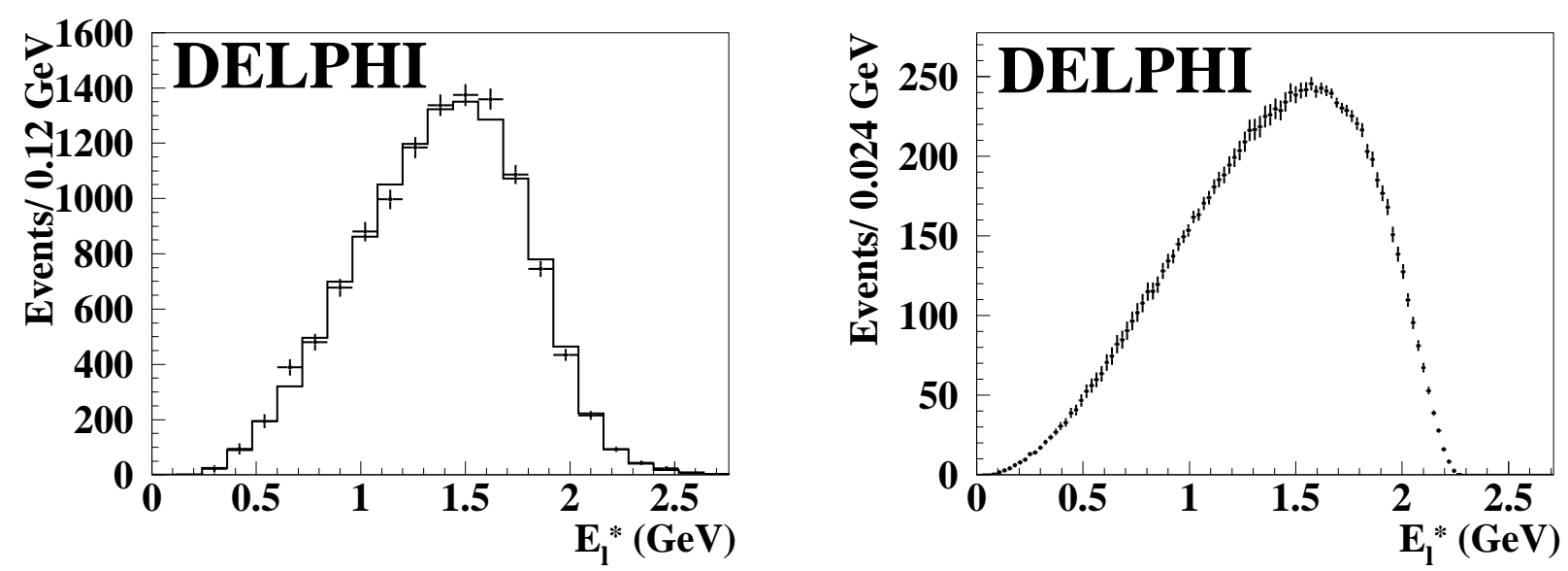

Figure 13: The background subtracted $E_{\ell}^{*}$ spectrum (left) for the selected signal sample and the unfolded lepton energy spectrum (right). 
The first, second and third moments, $M_{1}^{\ell}, M_{2}^{\prime \ell}$ and $M_{3}^{\prime \ell}$, have been computed. In order to reduce the systematic uncertainties, the second and third moments have been computed with respect to the average value.

\begin{tabular}{|c|c|c|c|}
\hline & $\begin{array}{c}M_{1}^{\ell} \\
(\mathrm{GeV})\end{array}$ & $\begin{array}{c}M_{2}^{\prime \ell} \\
(\mathrm{GeV})^{2}\end{array}$ & $\begin{array}{c}M_{3}^{\prime \ell} \\
(\mathrm{GeV})^{3}\end{array}$ \\
\hline value & 1.3782 & 0.1838 & -0.0301 \\
\hline stat. uncert. & \pm 0.0073 & \pm 0.0058 & \pm 0.0015 \\
\hline B species & \pm 0.0027 & $\mp 0.0017$ & $\mp 0.0005$ \\
B $\rightarrow$ D, D*, D ${ }^{* *} \ell \bar{\nu}_{\ell}$ & \pm 0.0010 & $\mp 0.0005$ & \pm 0.0001 \\
B fragmentation & \pm 0.0027 & $\mp 0.0020$ & $\mp 0.0007$ \\
B $\rightarrow$ X $_{u} \ell \bar{\nu}_{\ell}$ & \pm 0.0008 & \pm 0.0003 & $\mp 0.0001$ \\
e.m. radiation & \pm 0.0035 & $\mp 0.0001$ & $\mp 0.0004$ \\
\hline Bkg modelling & \pm 0.0026 & $\mp 0.0011$ & $\mp 0.0005$ \\
B direction reconstruction & \pm 0.0027 & $\mp 0.0018$ & $\mp 0.0006$ \\
B energy reconstruction & \pm 0.0027 & \pm 0.0011 & $\mp 0.0003$ \\
B mass cut & \pm 0.0051 & $\mp 0.0031$ & $\mp 0.0017$ \\
Unfolding & \pm 0.0031 & $\mp 0.0028$ & \pm 0.0029 \\
\hline Tot. syst. & \pm 0.0092 & \pm 0.0055 & \pm 0.0036 \\
\hline
\end{tabular}

Table 14: Corrected lepton moments and sources of systematic uncertainties.

The statistical correlation matrix for these three moments is given in Appendix B.

In order to relate the measured moments to those computed for a $\mathrm{B}$ meson, some corrections need to be applied.

Firstly the effect of radiation needs to be corrected for. This was done using correction factors computed separately for electrons and muons following ref. [41] and gives a shift of $+7.0 \mathrm{MeV},-0.2 \times 10^{-3} \mathrm{GeV}^{2}$ and $-0.7 \times 10^{-3} \mathrm{GeV}^{3}$ on the first, second and third moments, respectively. Half of these shifts have been used as an estimation of the systematic uncertainty on this correction. Since $e^{+} e^{-} \rightarrow b \bar{b}$ events at LEP result in the production of an admixture of $b$-hadron species, a correction factor accounting for the bias due to the semileptonic decays of the heavier $b$-hadrons was applied, using the fraction of $\overline{\mathrm{B}_{\mathrm{s}}^{0}}$ and of $b$-baryon left in the selected sample according to the simulation prediction. The uncertainty in the prediction is considered in the systematic uncertainty evaluation. Finally, the presence of $b \rightarrow \mathrm{X}_{u} \ell \bar{\nu}_{\ell}$ decays results in a similar bias of the lepton spectrum toward higher energies, due to the larger phase space of this decay compared to $b \rightarrow \mathrm{X}_{c} \ell \bar{\nu}_{\ell}$. This was also corrected for. Results, after corrections, are given in Table 14 .

Several sources of systematic uncertainties have been investigated and the results are summarized in Table 14. The sources related to the modelling used in the simulation are: the fractions of the different $\mathrm{D}$ species in the decays, the different $\mathrm{B}$ species and the $b$ fragmentation function. For the central value of the moments the same branching fractions for $\mathrm{B} \rightarrow \mathrm{D}, \mathrm{D}^{*}, \mathrm{D}^{* *}$ as in Section 4 , the $b$-hadron fractions from reference [21] and the results of reference [42] for the $b$-hadron fragmentation distribution have been used, respectively. The variations quoted therein have been used for evaluating the systematic uncertainties reported in Table 14 .

The uncertainty related to the background modelling has been evaluated by changing the simulation prediction for the cascade decays within the uncertainties of the branching ratios given in [21] and changing the misidentification efficiency according to Sections 2.2 and 2.3. It has also been checked by comparing the results obtained by using the background shape as extracted from the anti-tagged data with that using the prediction from the simulation. 
The uncertainty due to the $\mathrm{B}$ reconstruction accounts for variations in the $\mathrm{B}$ reconstructed energy and direction.

The uncertainty related to the unfolding procedure was evaluated by varying the reweighting function used in the fit and the binning.

The stability of the results with respect to changes in the selection cut applied on the VSEP variable and reconstructed B mass have been checked. Changes of the VSEP cut inducing variations of the accepted statistics up to a factor of 1.5 and of the signal purity over a range from $76 \%$ to $89 \%$ have been considered and found to give results stable within their statistical uncertainty. The minimum value of the $\mathrm{B}$ mass required has been moved between 2 and $5 \mathrm{GeV}$, with a corresponding change of purity between $71 \%$ and $86 \%$. The maximum variation with respect to the central value has been used as an estimation of the systematic uncertainty in the agreement between data and simulation on the B mass reconstruction.

Results obtained separately on the electron sample and the muon sample have been compared. The difference in the first moments amounts to $20 \pm 13 \pm 3 \mathrm{MeV}$, where the first uncertainty is statistical and the second is the uncorrelated systematic uncertainty on the background subtraction. The difference expected from simulation is $7 \mathrm{MeV}$. The differences on the second and third moments are fully compatible with the statistical uncertainty.

\section{$6 \quad$ Interpretation of the results}

A $\chi^{2}$ fit to the three leptonic $\left(M_{1}^{\ell}, M_{2}^{\prime \ell}\right.$ and $M_{3}^{\prime \ell}$, see Table 14) and three hadronic mass moments $\left(M_{1}^{H}, M_{2}^{\prime H}\right.$ and $M_{3}^{\prime H}$, see Tables 12 and 13) has been performed, using two theoretical frameworks. In the fit we also impose additional constraints derived from independent determinations. We follow the framework presented in [7], updated with the new results discussed above, and using recent calculations given in [43]. Parallel fits have also been performed by other groups using several frameworks [44].

In the kinetic mass scheme, we fit the full set of parameters: $m_{b}(1 \mathrm{GeV}), m_{c}(1 \mathrm{GeV})$, $\mu_{\pi}^{2}$ and $\tilde{\rho}_{D}^{3}$. Expressions relating moments and these parameters can be found in [7]. We impose $\mu_{G}^{2}=0.35 \pm 0.07 \mathrm{GeV}^{2}$ and $\rho_{L S}^{3}=-0.15 \pm 0.10 \mathrm{GeV}^{3}$ [43]. Two mass constraints have also been applied: $m_{b}(1 \mathrm{GeV})=4.61 \pm 0.17 \mathrm{GeV} / c^{2}$ and $m_{c}(1 \mathrm{GeV})=1.14 \pm 0.10 \mathrm{GeV} / c^{2}$ as derived from the values quoted in [45], which were given using a different renormalization scheme. Results are obtained for $\alpha_{s}\left(m_{b}\right)=0.22 \pm 0.04^{8}$ and are shown in Table 15 . In order to study the effect of the bounds on $m_{b, c}$ introduced, the fit has been repeated without these constraints. Results are shown in Table 16. Theoretical uncertainties have been evaluated following the procedure explained in [43]; namely $20 \%$ (30\%) errors have been assumed for terms corresponding to $1 / m_{b}^{2}\left(1 / m_{b}^{3}\right)$ corrections and adding in quadrature variations corresponding to the uncertainty on $\alpha_{s}\left(m_{b}\right)$. Corresponding theoretical uncertainties attached to $m_{b}$ and $m_{c}$ are due to those on $\alpha_{s}\left(m_{b}\right)$.

The central values of the heavy quark masses are in agreement with independent determinations $[46,47]$. The difference between the values of the two heavy quark masses, which are highly correlated, is $m_{b}(1 \mathrm{GeV})-m_{c}(1 \mathrm{GeV})=3.422 \pm 0.034 \pm 0.028 \mathrm{GeV} / c^{2}$ $\left(3.382 \pm 0.051 \pm 0.087 \mathrm{GeV} / c^{2}\right.$ if no constraint on the quark masses is imposed).

In the approach based on pole masses [48], the fit extracts $\bar{\Lambda}, \lambda_{1}, \lambda_{2}, \rho_{1}$ and $\rho_{2}$. We fix $\mathcal{T}_{i}=0.0 \mathrm{GeV}^{3}$ and impose two constraints on $M_{B^{*}}-M_{B}$ and $M_{D^{*}}-M_{D}$ which effectively reduces by two the number of free parameters. The results are given in Table 17.

\footnotetext{
${ }^{8}$ For hadron moments a value of $\alpha_{s}\left(m_{b}\right)=0.3 \pm 0.1$ has been used to account for missing terms in theoretical expressions.
} 


\begin{tabular}{|l|ccccc|}
\hline Fit & $\begin{array}{c}\text { Fit } \\
\text { Parameter }\end{array}$ & $\begin{array}{c}\text { Fit } \\
\text { Values }\end{array}$ & $\begin{array}{c}\text { Syst. } \\
\text { moments }\end{array}$ & $\begin{array}{c}\text { Syst. } \\
\text { theory }\end{array}$ & \\
\hline$m_{b}(1 \mathrm{GeV})$ & 4.591 & \pm 0.062 & \pm 0.039 & \pm 0.005 & $\mathrm{GeV} / c^{2}$ \\
$m_{c}(1 \mathrm{GeV})$ & 1.170 & \pm 0.093 & \pm 0.055 & \pm 0.005 & $\mathrm{GeV} / c^{2}$ \\
$\mu_{\pi}^{2}(1 \mathrm{GeV})$ & 0.399 & \pm 0.048 & \pm 0.034 & \pm 0.087 & $\mathrm{GeV}^{2}$ \\
$\tilde{\rho}_{D}^{3}$ & 0.053 & \pm 0.017 & \pm 0.011 & \pm 0.026 & $\mathrm{GeV}^{3}$ \\
\hline
\end{tabular}

Table 15: Results of the fit in the $m_{b}(\mu), m_{c}(\mu)$ and $\mu_{\pi}^{2}(\mu)$ formalism.

\begin{tabular}{|l|ccccc|}
\hline Fit & $\begin{array}{c}\text { Fit } \\
\text { Parameter }\end{array}$ & $\begin{array}{c}\text { Fit } \\
\text { Values }\end{array}$ & $\begin{array}{c}\text { Syst. } \\
\text { Uncertainty }\end{array}$ & $\begin{array}{c}\text { Syst. } \\
\text { theory }\end{array}$ & \\
\hline$m_{b}(1 \mathrm{GeV})$ & 4.67 & \pm 0.11 & \pm 0.19 & \pm 0.03 & $\mathrm{GeV} / c^{2}$ \\
$m_{c}(1 \mathrm{GeV})$ & 1.29 & \pm 0.17 & \pm 0.27 & \pm 0.04 & $\mathrm{GeV} / c^{2}$ \\
$\mu_{\pi}^{2}(1 \mathrm{GeV})$ & 0.41 & \pm 0.05 & \pm 0.04 & \pm 0.08 & $\mathrm{GeV}$ \\
$\tilde{\rho}_{D}^{3}$ & 0.05 & \pm 0.02 & \pm 0.01 & \pm 0.03 & $\mathrm{GeV}^{3}$ \\
\hline
\end{tabular}

Table 16: Results of the fit in the $m_{b}(\mu), m_{c}(\mu)$ and $\mu_{\pi}^{2}(\mu)$ formalism, without contraints on $m_{b}(1 \mathrm{GeV})$ and $m_{c}(1 \mathrm{GeV})$. Values of the fitted masses correspond to $\overline{m_{b}}\left(\overline{m_{b}}\right)^{\overline{M S}}=4.31 \pm 0.20 \mathrm{GeV} / c^{2}$ and $\overline{m_{c}}\left(\overline{m_{c}}\right)^{\overline{M S}}=1.37 \pm 0.24 \mathrm{GeV} / c^{2}$.

Up to first order corrections in $\alpha_{s}$, parameters corresponding to non-perturbative QCD corrections, entering in the two approaches, are related:

$$
\mu_{\pi}^{2}=-\lambda_{1}-\frac{\mathcal{T}_{1}+3 \mathcal{T}_{2}}{m_{b}} ; \mu_{G}^{2}=3 \lambda_{2}+\frac{\mathcal{T}_{3}+3 \mathcal{T}_{4}}{m_{b}} ; \tilde{\rho}_{D}^{3}=\rho_{1} ; \rho_{L S}^{3}=3 \rho_{2} .
$$

The parameter $\bar{\Lambda}$ enters in the expression relating heavy quark and heavy meson masses, which, for pseudo-scalar mesons reads:

$$
M_{B}=m_{b}+\bar{\Lambda}+\frac{\mu_{\pi}^{2}-\mu_{G}^{2}}{2 m_{b}}+\frac{\tilde{\rho}_{D}^{3}+\rho_{L S}^{3}-\rho_{N L}^{3}}{4 m_{b}^{2}}+\mathcal{O}\left(\frac{1}{m_{b}^{3}}\right),
$$

where $\rho_{N L}^{3}$ corresponds to a linear combination of $\mathcal{T}_{1-4}$. Projections of the constraints from the six moments in the $m_{b}-\mu_{\pi}^{2}$ and $m_{b}-\tilde{\rho}_{D}^{3}$ planes are shown in Figure 14 and those in the $\bar{\Lambda}-\lambda_{1}$ and $\bar{\Lambda}-\rho_{1}$ planes in Figure 15. The $\chi^{2} /$ n.d.f. of the fits is 0.4 and 0.2 in the two formulations. Since the contributions proportional to $\rho_{L S}^{3}$ in the moment expressions are numerically suppressed, the fit is only marginally sensitive to its size and the result is determined by the constraint applied. By removing this, the fit would give $\rho_{L S}^{3}=$ $-0.4 \pm 0.4 \mathrm{GeV}^{3}$. In contrast, the value of the leading $1 / \mathrm{m}_{b}^{3}$ correction (parametrised by $\tilde{\rho}_{D}^{3}$ ) can be determined with satisfactory accuracy and its range agrees with theoretical expectations [6]. These Figures illustrate the importance of the second hadronic moment to determine $\mu_{\pi}^{2}(1 \mathrm{GeV})$ and of the second and third hadronic moments to extract $\tilde{\rho}_{D}^{3}$.

In Tables 15 and 17, the first column of systematic uncertainties corresponds to systematics on moments; correlated errors between the different moments have been included in the fit. The second column is due to systematics from theory. For the kinetic mass formalism we propagate the uncertainty on $\alpha_{s}$ and follow the suggestions of [43] to account for missing corrections. 


\begin{tabular}{|l|rcccc|}
\hline Fit & $\begin{array}{c}\text { Fit } \\
\text { Values }\end{array}$ & $\begin{array}{c}\text { Fit } \\
\text { Uncertainty }\end{array}$ & $\begin{array}{c}\text { Syst. } \\
\text { moments }\end{array}$ & $\begin{array}{c}\text { Syst. } \\
\text { theory }\end{array}$ & \\
\hline$\Lambda$ & 0.601 & \pm 0.065 & \pm 0.061 & \pm 0.05 & $\mathrm{GeV}$ \\
$\lambda_{1}$ & -0.252 & \pm 0.054 & \pm 0.018 & \pm 0.07 & $\mathrm{GeV}^{2}$ \\
$\lambda_{2}$ & 0.117 & \pm 0.003 & \pm 0.002 & \pm 0.00 & $\mathrm{GeV}^{2}$ \\
$\rho_{1}$ & 0.032 & \pm 0.021 & \pm 0.010 & \pm 0.04 & $\mathrm{GeV}^{3}$ \\
\hline$\rho_{2}$ & 0.085 & \pm 0.011 & \pm 0.017 & \pm 0.21 & $\mathrm{GeV}^{3}$ \\
\hline
\end{tabular}

Table 17: Results of the fit in the $\bar{\Lambda}-\lambda_{1}$ formalism.

For the $\bar{\Lambda}-\lambda_{1}$ formalism we include in the theory systematics the effect of $\mathcal{T}_{i}=(0.0 \pm$ $0.50)^{3}, \alpha_{s}=0.22 \pm 0.04$ and we also estimate the effect of the missing corrections to third moments as explained in [7].
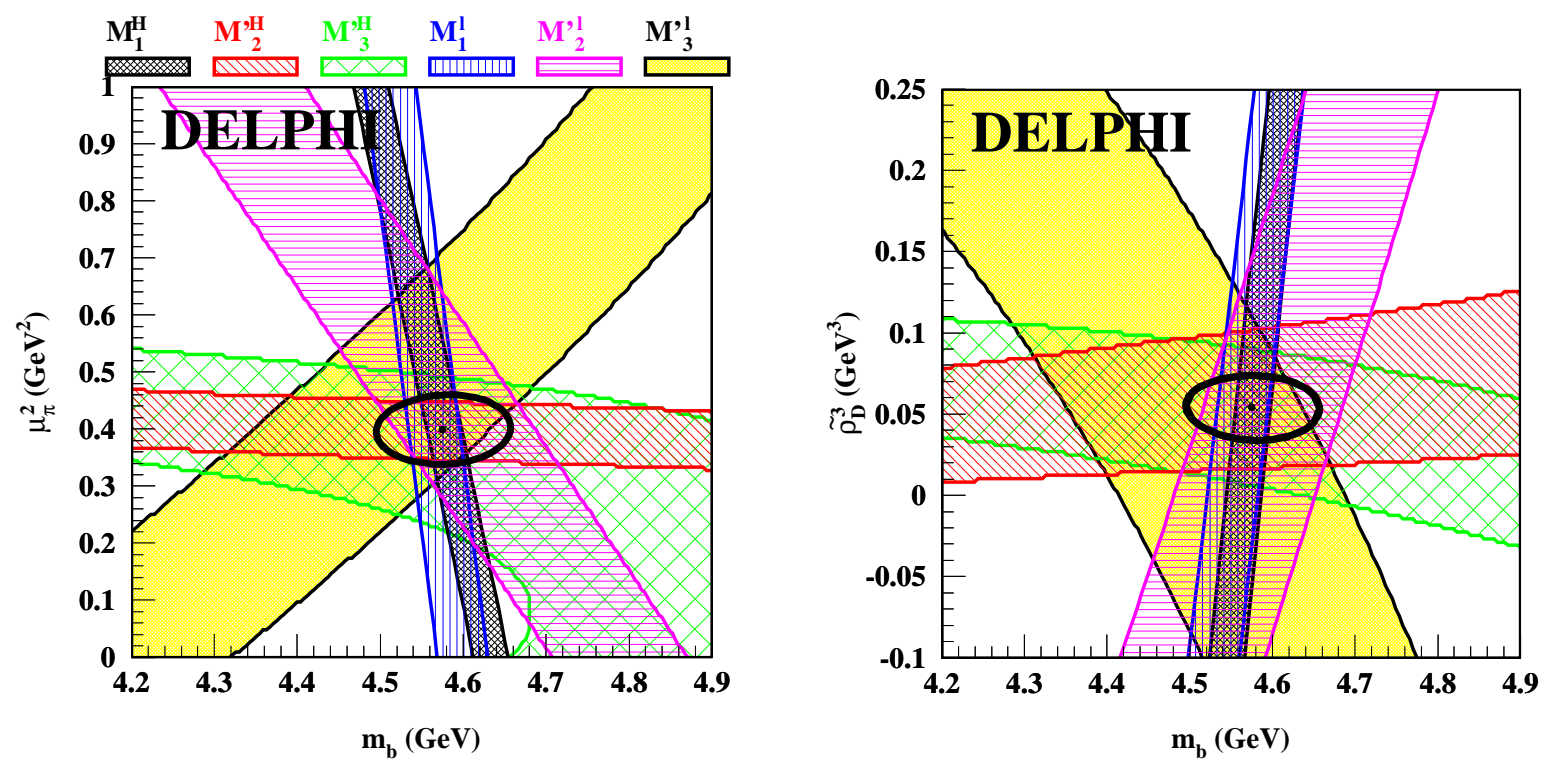

Figure 14: The projection of the constraints of the six measured moments on the $m_{b}(1 \mathrm{GeV})-\mu_{\pi}^{2}(1 \mathrm{GeV})$ (left) and $m_{b}(1 \mathrm{GeV})-\tilde{\rho}_{D}^{3}$ (right) planes. The bands correspond to the total measurement accuracy and are given by keeping all the other parameters at their central values. The ellipses represent the $1 \sigma$ contours and include correlations between the parameters.

\subsection{Implications for $\left|V_{c b}\right|$}

The value of $\left|\mathrm{V}_{c b}\right|$ obtained from the total semileptonic decay width depends on the OPE parameters extracted above. We discuss now the implications of our results for $\left|\mathrm{V}_{c b}\right|$, using the input parameters given in Table 18, which correspond to measurements obtained at LEP.

The determination of $\left|\mathrm{V}_{c b}\right|$ and the contributions of the various parameters in the kinetic mass scheme are described in [8]. This approach has been preferred to the pole 



Figure 15: The projection of the constraints of the six measured moments on the $\bar{\Lambda}-\lambda_{1}$ (left) and $\bar{\Lambda}-\rho_{1}$ (right) planes. The bands correspond to the total measurement accuracy and are given by keeping all the other parameters at their central values. The ellipses represent the $1 \sigma$ contours and include correlations between the parameters.

\begin{tabular}{|c|c|c|}
\hline Measurement & Value & Reference \\
\hline$b$-hadron lifetime & $1.573 \pm 0.007 \mathrm{ps}$ & HFAG Winter 2003 [38] \\
$\mathrm{BR}\left(b \rightarrow X \ell^{-} \nu\right)$ & $(10.65 \pm 0.23) \%$ & LEPEWWG/2003-01 [49] \\
$\mathrm{BR}\left(b \rightarrow \mathrm{X}_{u} \ell^{-} \nu\right)$ & $(0.17 \pm 0.05) \%$ & PDG 2002 [21] \\
\hline
\end{tabular}

Table 18: Input values, obtained at LEP, used for the determination of $\left|V_{c b}\right|$.

mass scheme as it does not rely on an expansion in $1 / m_{c}$ and also because corrections contributing at order $1 / m_{b}^{3}$ have been fixed by experiment. Using the expression of $\left|\mathrm{V}_{c b}\right|$ quoted in $[2]^{9}$, it gives:

$$
\left|\mathrm{V}_{c b}\right|=0.0421 \times\left(1 \pm 0.014_{\text {meas. }} \pm 0.014_{\text {fit }} \pm 0.015_{\text {th. }}\right),
$$

where the first uncertainty reflects the accuracy on the semileptonic width determination. This experimental uncertainty, corresponding to LEP-alone results, can be reduced to $\pm 1 \%$ by including recent measurements obtained at B-factories which gave [38]:

$\operatorname{BR}\left(b \rightarrow X \ell^{-} \bar{\nu}_{\ell}\right)=(10.73 \pm 0.28) \%$, in agreement with the LEP measurement and having a similar accuracy. The second uncertainty, given in Equation (21), corresponds to uncertainties from the fit of the parameters, obtained in Table 15 and not including the theoretical uncertainties given in the last column. The third uncertainty has been taken from the result given in [2].

\footnotetext{
${ }^{9}$ We have used the Equation (10) given in [2].
} 


\section{Conclusions}

Production characteristics of $\mathrm{D}^{* *}$ mesons in $b$-hadron semileptonic decays have been studied using exclusively reconstructed decay channels.

The total production fraction has been measured to be:

$$
\mathrm{BR}\left(\overline{\mathrm{B}_{\mathrm{d}}^{0}} \rightarrow \mathrm{D}^{* *} \ell^{-} \bar{\nu}_{\ell}\right)=(2.7 \pm 0.7 \pm 0.2) \%
$$

Decay final states are dominated by the $\mathrm{D}^{(*)} \pi$ channel and no-evidence for a signal in channels with two pions has been obtained:

$$
\begin{gathered}
\mathrm{BR}\left(b \rightarrow \mathrm{D}^{0} \pi^{+} \pi^{-} \ell^{-} \bar{\nu}_{\ell}\right)=\mathrm{BR}\left(b \rightarrow \mathrm{D}^{+} \pi^{+} \pi^{-} \ell^{-} \bar{\nu}_{\ell}\right)<0.18 \% \text { at } 90 \% \text { C.L. } \\
\mathrm{BR}\left(b \rightarrow \mathrm{D}^{*+} \pi^{+} \pi^{-} \ell^{-} \bar{\nu}_{\ell}\right)<0.13 \% \text { at } 90 \% \text { C.L. }
\end{gathered}
$$

The dominant contributing channel is the broad $\mathrm{D}_{1}^{*}$ whose mass and total width have been measured to be:

$$
\begin{aligned}
m_{D_{1}^{*}} & =2445 \pm 34 \pm 10 \mathrm{MeV} / c^{2} \\
\Gamma_{D_{1}^{*}} & =234 \pm 74 \pm 25 \mathrm{MeV} / c^{2} .
\end{aligned}
$$

Broad $\mathrm{D} \pi$ final states favour a production which is maximum close to threshold, as is expected from non-resonant production, but the present statistics do not allow this feature to be firmly established.

Moments of the hadronic mass distribution corresponding to $\mathrm{D}^{* *}$ states in $b$-hadron semileptonic decays have been measured:

$$
\begin{aligned}
& <m_{D^{* *}}>=2.483 \pm 0.033 \pm 0.033 \mathrm{GeV} / c^{2} \\
& <m_{D^{* *}}^{2}>=6.22 \pm 0.16 \pm 0.15\left(\mathrm{GeV} / c^{2}\right)^{2} \\
& <m_{D^{* *}}^{4}>=40.1 \pm 2.0 \pm 1.7\left(\mathrm{GeV} / c^{2}\right)^{4} \\
& <m_{D^{* *}}^{6}>=271 \pm 21 \pm 16\left(\mathrm{GeV} / c^{2}\right)^{6} \\
& <m_{D^{* *}}^{8}>=(19.3 \pm 2.1 \pm 1.4) 10^{2}\left(\mathrm{GeV} / c^{2}\right)^{8} \\
& <m_{D^{* *}}^{10}>=(14.7 \pm 2.0 \pm 1.2) 10^{3}\left(\mathrm{GeV} / c^{2}\right)^{10}
\end{aligned}
$$

Using these results and world averaged measurements for the exclusive $b$-hadron semileptonic decay fractions into a $\mathrm{D}$ or a $\mathrm{D}^{*}$ meson, various moments of the full hadronic mass distribution have been obtained in Section 4.

Moments of the lepton energy spectrum in semileptonic B decays have also been measured as:

$$
\begin{gathered}
<E_{\ell}^{*}>=1.3782 \pm 0.0073 \pm 0.0092 \mathrm{GeV} \\
<\left(E_{\ell}^{*}-<E_{\ell}^{*}>\right)^{2}>=0.1838 \pm 0.0058 \pm 0.0055 \mathrm{GeV}^{2} \\
<\left(E_{\ell}^{*}-<E_{\ell}^{*}>\right)^{3}>=-0.0301 \pm 0.0015 \pm 0.0036 \mathrm{GeV}^{3} .
\end{gathered}
$$

These results are interpreted in terms of constraints on the values of heavy quark masses, of the $b$-quark kinetic energy and of the parameters contributing at order $1 / m_{b}^{3}$ in theoretical expressions for the $b$-hadron semileptonic partial decay width. The values obtained are:

$$
\begin{aligned}
m_{b}(1 \mathrm{GeV}) & =4.591 \pm 0.062 \pm 0.039 \pm 0.005 \mathrm{GeV} / c^{2} \\
m_{c}(1 \mathrm{GeV}) & =1.170 \pm 0.093 \pm 0.055 \pm 0.005 \mathrm{GeV} / c^{2} \\
\mu_{\pi}^{2}(1 \mathrm{GeV}) & =0.399 \pm 0.048 \pm 0.034 \pm 0.087 \mathrm{GeV}^{2} \\
\tilde{\rho}_{D}^{3} & =0.053 \pm 0.017 \pm 0.011 \pm 0.026 \mathrm{GeV}^{3}
\end{aligned}
$$


and include corrections at order $1 / m_{b}^{3}$.

Using these results, and inclusive measurements of the $b$-hadron lifetime and semileptonic branching fraction obtained at LEP, an accurate determination of the value of the $\left|\mathrm{V}_{c b}\right|$ element has been obtained:

$$
\left|\mathrm{V}_{c b}\right|=0.0421 \times\left(1 \pm 0.014_{\text {meas. }} \pm 0.014_{\text {fit }} \pm 0.015_{\text {th. }}\right) .
$$

The first uncertainty becomes $\pm 1 \%$ if measurements of $\overline{\mathrm{B}_{\mathrm{d}}^{0}}$ and $\mathrm{B}^{-}$lifetime and semileptonic decay rates, obtained at the $\Upsilon(4 \mathrm{~S})$, are included.

\section{Acknowledgements}

We would like to thank P. Gambino and N. Uraltsev for helpful discussions during this work and for providing us with the necessary theoretical expressions. We had also useful discussions on the properties of the non-resonant $\mathrm{D}^{(*)} \pi$ component with A. Le Yaouanc. I. Bigi is warmly thanked for his guidance through the theoretical formalism.

We are greatly indebted to our technical collaborators, to the members of the CERNSL Division for the excellent performance of the LEP collider, and to the funding agencies for their support in building and operating the DELPHI detector. We acknowledge in particular the support of Austrian Federal Ministry of Education, Science and Culture, GZ 616.364/2-III/2a/98, FNRS-FWO, Flanders Institute to encourage scientific and technological research in the industry (IWT), Belgium, FINEP, CNPq, CAPES, FUJB and FAPERJ, Brazil, Czech Ministry of Industry and Trade, GA CR 202/99/1362,

Commission of the European Communities (DG XII), Direction des Sciences de la Matière, CEA, France, Bundesministerium für Bildung, Wissenschaft, Forschung und Technologie, Germany, General Secretariat for Research and Technology, Greece,

National Science Foundation (NWO) and Foundation for Research on Matter (FOM), The Netherlands,

Norwegian Research Council,

State Committee for Scientific Research, Poland, SPUB-M/CERN/PO3/DZ296/2000, SPUB-M/CERN/PO3/DZ297/2000, 2P03B 10419 and 2P03B 69 23(2002-2004)

JNICT-Junta Nacional de Investigação Científica e Tecnológica, Portugal, Vedecka grantova agentura MS SR, Slovakia, Nr. 95/5195/134, Ministry of Science and Technology of the Republic of Slovenia, CICYT, Spain, AEN99-0950 and AEN99-0761, The Swedish Natural Science Research Council, Particle Physics and Astronomy Research Council, UK, Department of Energy, USA, DE-FG02-01ER41155, EEC RTN contract HPRN-CT-00292-2002. 


\section{A Error matrices for hadronic mass moments}

In these matrices, which refer to moments $M_{1}^{H}, M_{2}^{H}, M_{3}^{H}, M_{4}^{H}, M_{5}^{H}, M_{2}^{\prime H}, M_{3}^{\prime H}$, $M_{4}^{\prime H}$ and $M_{5}^{\prime H}$ given in this order, the diagonal elements are the errors and non-diagonal elements correspond to correlation coefficients.

Statistical error matrix:

$$
\left(\begin{array}{ccccccccc}
0.0455 & 0.947 & 0.865 & 0.812 & 0.921 & 0.907 & 0.725 & 0.724 & 0.698 \\
& 0.232 & 0.978 & 0.951 & 0.996 & 0.994 & 0.904 & 0.899 & 0.878 \\
& & 1.29 & 0.994 & 0.992 & 0.994 & 0.973 & 0.969 & 0.956 \\
& & & 7.92 & 0.974 & 0.975 & 0.990 & 0.990 & 0.983 \\
& & & & 1080 . & 0.998 & 0.936 & 0.933 & 0.916 \\
& & & & & 0.176 & 0.943 & 0.936 & 0.918 \\
& & & & & & 0.736 & 0.998 & 0.993 \\
& & & & & & & 4.53 & 0.998 \\
& & & & & & & & 27.0
\end{array}\right)
$$

Error matrix for systematics:

$$
\left(\begin{array}{ccccccccc}
0.0896 & 0.981 & 0.949 & 0.905 & 0.863 & 0.946 & 0.655 & 0.664 & 0.540 \\
& 0.273 & 0.981 & 0.945 & 0.908 & 0.990 & 0.749 & 0.747 & 0.630 \\
& & 1.09 & 0.987 & 0.967 & 0.980 & 0.848 & 0.853 & 0.758 \\
& & & 5.42 & 0.995 & 0.952 & 0.910 & 0.918 & 0.844 \\
& & & & 32.3 & 0.920 & 0.937 & 0.950 & 0.891 \\
& & & & & 0.161 & 0.801 & 0.789 & 0.680 \\
& & & & & & 0.318 & 0.992 & 0.974 \\
& & & & & & & 2.06 & 0.986 \\
& & & & & & & & 11.7
\end{array}\right)
$$

Total error matrix:

$$
\left(\begin{array}{ccccccccc}
0.100 & 0.945 & 0.844 & 0.759 & 0.440 & 0.872 & 0.533 & 0.544 & 0.481 \\
& 0.358 & 0.966 & 0.914 & 0.664 & 0.984 & 0.763 & 0.765 & 0.712 \\
& & 1.69 & 0.987 & 0.778 & 0.987 & 0.900 & 0.903 & 0.866 \\
& & & 9.59 & 0.820 & 0.957 & 0.954 & 0.959 & 0.934 \\
& & & & 1080 & 0.755 & 0.870 & 0.860 & 0.851 \\
& & & & & 0.239 & 0.853 & 0.849 & 0.804 \\
& & & & & & 0.802 & 0.997 & 0.990 \\
& & & & & & & 4.98 & 0.996 \\
& & & & & & & & 29.5
\end{array}\right)
$$




\section{B Error matrices for lepton energy moments}

In these matrices, which refer to moments $M_{1}^{l}, M_{2}^{\prime \ell}$ and $M_{3}^{\prime \ell}$ given in this order, the diagonal elements are the errors and non-diagonal elements correspond to correlation coefficients.

Statistical error matrix:

$$
\left(\begin{array}{rrr}
0.0073 & -0.6041 & -0.3435 \\
& 0.0058 & -0.5381 \\
& & 0.0015
\end{array}\right)
$$

Error matrix for systematics:

$$
\left(\begin{array}{rrr}
0.0092 & -0.7823 & -0.2427 \\
& 0.0055 & 0.0342 \\
& & 0.0036
\end{array}\right)
$$

Total error matrix:

$$
\left(\begin{array}{rrr}
0.0118 & -0.6942 & -0.2578 \\
& 0.0080 & -0.1286 \\
& & 0.0039
\end{array}\right)
$$




\section{References}

[1] I. Bigi, M. Shifman and N.G. Uraltsev, Ann. Rev. Nucl. Part. Sci. 47 (1997) 591 and references therein.

[2] D. Benson, I.I. Bigi, T. Mannel and N. Uraltsev, Nucl. Phys. B665 (2003) 367, [hep-ph/0302262].

[3] A. Sirlin, Nucl. Phys. B71 (1964) 29, and Rev. Mod. Phys. 50 (1978) 573. Erratum-ibid. 50 (1978) 905.

[4] J. Chay, H. Georgi and B. Grinstein, Phys. Lett. B247 (1990) 399.

[5] I. I. Y. Bigi, N. G. Uraltsev and A. I. Vainshtein, Phys. Lett. B293 (1992) 430. Erratum-ibid. B297 (1993) 477, [hep-ph/9207214].

[6] N. Uraltsev, Phys. Lett. B545 (2002) 337 [hep-ph/0111166].

[7] M. Battaglia et al. Phys. Lett. B556 (2003) 41, [hep-ph/0210319].

[8] N. Uraltsev, Mod. Phys. Lett. A17 (2002) 2317, [hep-ph/0210413].

[9] N.E. Adam et al. (CLEO Collaboration), Phys. Rev. D67 (2003) 032001.

[10] D. Cronin-Hennessy et al. (CLEO Collaboration), Phys. Rev. Lett. 87 (2001) 251808.

[11] S.E. Csorna et al. (CLEO Collaboration), Phys. Rev. D70 (2004) 032002 [hepex/0403052].

[12] B. Aubert et al. (BaBar Collaboration), BABAR-CONF-02/029, SLAC-PUB9314, [hep-ex/0207084].

[13] B. Aubert et al. (BaBar Collaboration), Phys. Rev. D69 (2004) 111103, [hepex/0403031].

[14] B. Aubert et al. (BaBar Collaboration), Phys. Rev. D69 (2004) 111104, [hepex/0403030].

[15] K. Abe et al. (Belle Collaboration), BELLE-CONF-0474, [hep-ex/0409015].

[16] K. Abe et al. (Belle Collaboration), BELLE-CONF-0426, [hep-ex/0408139].

[17] D. Acosta et al. (CDF Collaboration), Phys. Rev. D71 (2005) 051103, [hepex/0502003].

[18] H. Albrecht et al., (ARGUS Collaboration), Z. Phys. C57 (1993) 533.

[19] D. Buskulic et al., (ALEPH Collaboration), Z. Phys. C73 (1997) 601.

[20] J.L. Rosner, Comments Nucl. Part. Phys. 16 (1986) 109.

[21] K. Hagiwara et al., (Particle Data Group), Phys. Rev. D66 (2002) 010001.

[22] A. Anastassov et al., (CLEO Collaboration), Phys. Rev. Lett. 80 (1998) 4127.

[23] S. Godfrey and N. Isgur, Phys. Rev. D32 (1985) 189.

[24] J.L. Goity and W. Roberts, Phys. Rev. D51 (1995) 3459.

[25] P. Abreu et al., (DELPHI Collaboration), Phys. Lett. B475 (2000) 407.

[26] T. Sjöstrand, Comp. Phys. Comm. 82 (1994) 74.

[27] P. Abreu et al., (DELPHI Collaboration), Nucl. Instr. and Meth. A378 (1996) 57, Erratum-ibid. A396 (1997) 281.

[28] J. Abdallah et al., (DELPHI Collaboration), Eur. Phys. J. C32 (2004) 185.

[29] J. Abdallah et al., (DELPHI Collaboration), Eur. Phys. J. C33 (2004) 213, [hepex/0401023]

[30] R. Barlow and C. Beeston, Comp. Phys. Comm. 77 (1993) 219.

[31] R. Barate et al., (ALEPH Collaboration), Eur. Phys. J. C4 (1998) 387.

[32] B. Aubert et al., (BaBar Collaboration), Phys. Rev. D68 (2003) 092001, [hepex/0305003]. 
[33] Combined results on $b$-hadron production rates, lifetimes, oscillations and semileptonic decays (ALEPH, CDF, DELPHI, L3, OPAL, SLD), CERN-EP/2001-050, [hep-ex/0112028].

[34] H. Albrecht et al., (ARGUS Collaboration), Phys. Lett. B232 (1989) 398;

P. Avery et al., (CLEO Collaboration), Phys. Rev. D41 (1990) 774, Phys. Lett. B331 (1994) 236 and Erratum-ibid. B342 (1995) 453.

[35] S. Anderson et al. (CLEO Collaboration), Nucl. Phys. A663 (2000) 647.

[36] K. Abe et al. (BELLE Collaboration), Phys. Rev. D69 (2004) 112002, [hepex/0307021].

[37] A. Anastassov et al. (CLEO Collaboration), Phys. Rev. D65 (2002) 032003.

[38] Heavy Flavour Averaging Group (http://www.slac.stanford.edu/xorg/hfag/).

[39] Z. Albrecht, M. Feindt, M. Moch, 'MACRIB- High Efficiency, High Purity Hadron Identification for DELPHI', DELPHI internal note, 99-150 RICH 95 (1999), [hepex/0111081].

[40] V. Blobel, OPAL technical Note TN361 (1996) [http://www-zeus.desy.de/ desler/blobel.html].

[41] D. Atwood and W. J. Marciano, Phys. Rev. D41 (1990) 1736.

[42] A Study of the $b$-Quark Fragmentation Function with the DELPHI Detector at LEP I, G. Barker et al. (DELPHI Collaboration), ICHEP2002, DELPHI note 2002-069-CONF-603.

[43] P. Gambino, N. Uraltsev, Eur. Phys. J. C34 (2004) 181.

[44] C.W. Bauer et al. Phys. Rev. D70 (2004) 094017, [hep-ph/0408002];

B. Aubert et al. (BaBar Collaboration), Phys. Rev. Lett. 93 (2004) 011803, [hepex/0404017]; O. Buchmüller and H. Flächer, [hep-ph/0507253].

[45] S. Eidelman et al., (Particle Data Group), Phys. Lett. B592 (2004) 1.

[46] Heavy Quarkonium Dynamics, A. Hoang, [hep-ph/0204299].

[47] K. Melnikov and A. Yelkhovsky, Phys. Rev. D59 (1999) 114009.

[48] A.F. Falk and M.E. Luke, Phys. Rev. D57 (1998) 424, [hep-ph/9708327].

[49] A combination of preliminary electroweak measurements and constraints on the Standard Model, CERN-EP/2003-091, [hep-ex/0312023]. 